\title{
苯并啡啶类生物碱及其衍生物合成研究进展
}

\author{
程 辟 $a$ 曾建国*,a,b

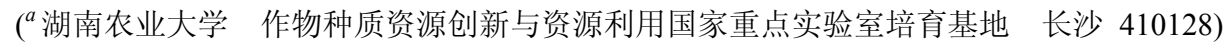 \\ ( ${ }^{b}$ 湖南农业大学 国家中药材生产(湖南)技术中心 长沙 410128)
}

\begin{abstract}
摘要 苯并啡啶类生物碱是一类广泛分布于罂栗科和芸香科植物中的天然含氮化合物, 具有显著的抗肿瘤和广谱抗菌 等多种生物活性. 基于苯并啡啶类生物碱的生源合成途径的特殊性对天然苯并啡啶类生物碱结构类型进行了分类. 综 述了自 1999 年以来天然苯并啡啶类生物碱及其衍生物的合成方法研究进展, 对各个合成路线的特点进行了讨论.

关键词 苯并啡啶类生物碱; 衍生物; 合成; 研究进展
\end{abstract}

\section{Progresses in Synthesis of Benzophenanthridine Alkaloids and Their Derivatives}

\author{
Cheng, $\mathrm{Pi}^{a} \quad$ Zeng, Jianguo ${ }^{*, a, b}$ \\ $\left({ }^{a}\right.$ Pre-State Key Laboratory for Germplasm Innovation and Utilization of Crop, Hunan Agricultural University, \\ Changsha 410128) \\ $\left({ }^{b}\right.$ National Chinese Medicinal Herbs (Hunan) Technology Center, Hunan Agricultural University, Hunan Agricultural \\ University, Changsha 410128)
}

\begin{abstract}
Benzophenanthridine alkaloids are a small family of natural $N$-containing compounds that widely distribute in papaveraceous and rutaceous plants and display obvious antitumor and antibacterial activities. In this review, the structure types of natural benzophenanthridine alkaloids are classified based on the particularity of biosynthetic pathway of this group of alkaloids. The synthetic methods of benzophenanthridine alkaloids and related derivatives from 1999 are reviewed with discussion on particularity of each synthetic route.
\end{abstract}

Keywords benzophenanthridine alkaloids; derivatives; synthesis; progresses

苯并啡啶类生物碱(benzophenanthridine alkaloids) 是植物生物碱类次生代谢产物中一个较小的结构类型, 常见于芸香科花椒属和罂粟科植物中 ${ }^{[1]}$. 这类生物碱在 1839 年已有首次报道, 一个世纪后两面针碱(Nitidine) 和花椒宁碱(Fagaronine)相继从芸香科植物中得到分离. 由于苯并啡啶类生物碱具有广泛的生理活性, 人们逐渐 认识到其重要的研究价值. 从 20 世纪 90 年代开始, 全 球多个研究小组针对天然苯并啡啶类生物碱的抗肿瘤、 抗菌活性和作用机制进行了系统的研究. 如血根碱 (Sanguinarine)和白屈菜红碱(Chelerythrine)具有显著的 广谱抗菌活性 ${ }^{[2]}$. 在相关终端产品方面最具代表性的是 德国 Phytobiotics 公司与湖南美可达生物资源有限公司 合作开发的替代抗生素饲料添加剂 Sangrovit ${ }^{[[3]}$ 以及美
国 Camas 公司开发的植物源杀菌剂 $\mathrm{QWEL}^{[4]}$. 其中 Sangrovit ${ }^{\circledR}$ 远销全球 70 多个国家和地区, 主要成分为来 自䔄粟科植物博落回中具有抗菌活性的血根碱等季铵 类本并啡啶生物碱 ${ }^{[5]}$. 此外，两面针碱和花椒宁碱被证 实具有抗肿瘤和抗病毒活性，能够作用于拓扑异构酶和 病毒的逆转录酶 ${ }^{[6]} .1997$ 年和 1999 年 Mackay ${ }^{[6]}$ 和 Ishikawa $^{[7]}$ 分别就具有抗肿瘤活性的苯并啡啶类生物碱 的全合成方法进行了文献综述. 由于天然苯并啡啶类生 物碱生源合成途径和化学结构的特殊性, 其母核结构取 代基的多样性受到了限制. 近十多年来, 苯并啡啶生物 碱基本骨架的高效合成方法得到了很大的发展，有效地 促进了这类生物碱的生物活性和构效关系研究. 本文将 基于天然苯并啡啶类生物碱的结构亚型类别、生源合成

* E-mail: ginkgo@world-way.net

Received April 6, 2012; revised May 1, 2012; published online May 11, 2012.

Project supported by the International Scientific and Technological Cooperation Program of State Ministry of Science and Technology (No. 2010DFA32810). 科技部国际科技合作计划(No. 2010DFA32810)资助项目. 
途径、基本骨架合成策略, 对 1999 年之后的相关文献进 行综述.

天然苯并啡啶类生物碱母核取代基主要为烷氧基 或羟基, 这与其生源合成途径的特殊性密切相关. 德国 $Z_{\text {Znk }}{ }^{[8]}$ 在苯并啡啶类生物碱生源合成途径方面做了非 常出色的研究. 该课题组通过愮粟科植物花菱草细胞培 养对大部分天然苯并啡啶类生物碱的生源合成途径和 相关生物合成酶进行了验证. 苯并啡啶类生物碱的生源 合成前体为苄基异喹啉生物碱( $S$ )-Reticuline (Scheme 1), 经多步酶催化反应后 $(S)$-Reticuline 转化为原阿片类生物 碱 Protopine. 在 P6H (Protopine 6-hydroxylase)的区域选 择性催化下, Protopine 6 位羟基化生成氮杂缩醛中间体 6-hydroxyprotopine. 氮杂缩醛中间体 6-hydroxyprotopine 发生分子内重排转化为二氢血根碱. 重排过程在植 物体内自发进行, 无需酶催化并且没有稳定的中间体存 在. 二氢血根碱 $\left(O_{4}\right.$-生物碱)在细胞色素 P450 氧化酶系 和 $O$-甲基转移酶系的催化下, 交替发生母核羟基化和
$O$-甲基化反应得到 $O_{5}$-和 $O_{6}$-生物碱的 5,6-氢化产物，随 后在 DBOX (Dihydrobenzophenathridine oxidase)的催化 下生成对应的全芳香化季铵类苯并啡啶生物碱. 本研究 室在对罂粟科血根碱来源植物博落回转录组数据(SRA: SRA048772)分析时发现，该植物不同器官部位中 $\mathrm{P} 6 \mathrm{H}$ 表达量的高低与血根碱含量高低密切相关, 因可以认为 P6H 是苯并啡定生物碱生源合成途径中关键酶之一, P6H 催化 Protopine 6 位差基化反应是苯并啡啶生物碱生 源合成途径中的关键步骤.

在 Ishikawa 对天然苯并啡啶类生物碱分类的基础 上, 我们依据生物碱母核的氧化程度将其分为两个类型 (Scheme 2): 部分氢化生物碱(partially hydrogenated base)和全芳香化生物碱(fully aromatized base), 其中部 分氢化生物碱又分为 $\mathrm{B}, \mathrm{C}$ 环氢化生物碱和 5,6-二氢生物 碱; 天然全芳香化生物碱又可以进一步分类为 $\mathrm{O}_{4}, \mathrm{O}_{5}$, $\mathrm{O}_{6}$ 三个亚类，下标数字为苯并啡啶类生物碱母核烷氧

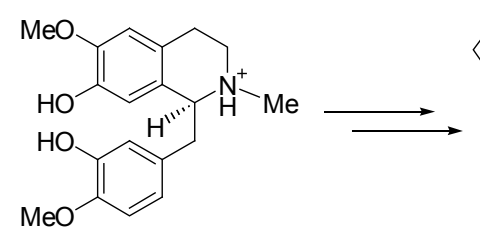

(S)-Reticuline<smiles></smiles>

Protopine

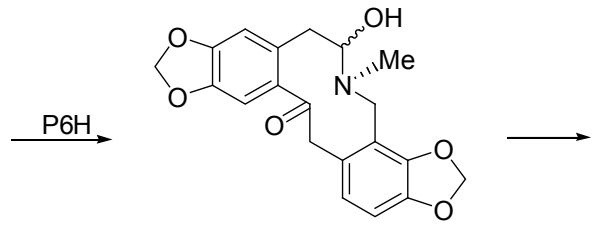

6-Hydroxyprotopine<smiles>CNC(=O)c1c(CCC=O)ccc2c1OCO2</smiles><smiles>COc1cc2c(cc1OC)OCO2</smiles>

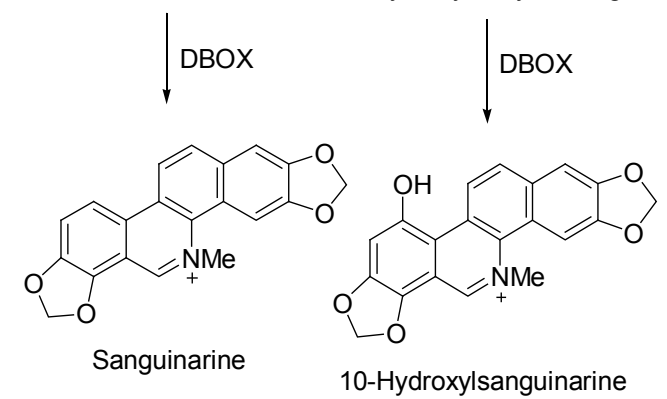

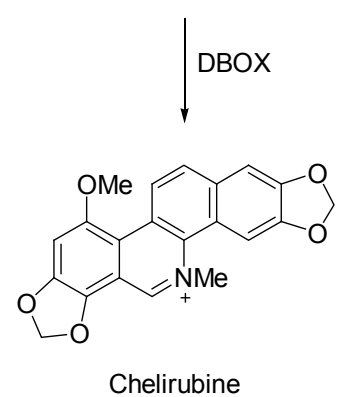

12-Hydroxyl-dihydrochelirubine Dihydromacarpine DBOX DBOX DBOX<smiles>COc1cc2c(c3cnc4c5cc6c(cc5c(O)cc4c13)OCO6)OCO2</smiles>

12-Hydroxylchelirubine

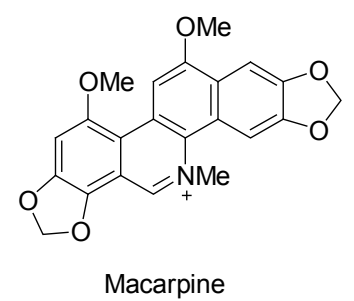

Scheme 1 


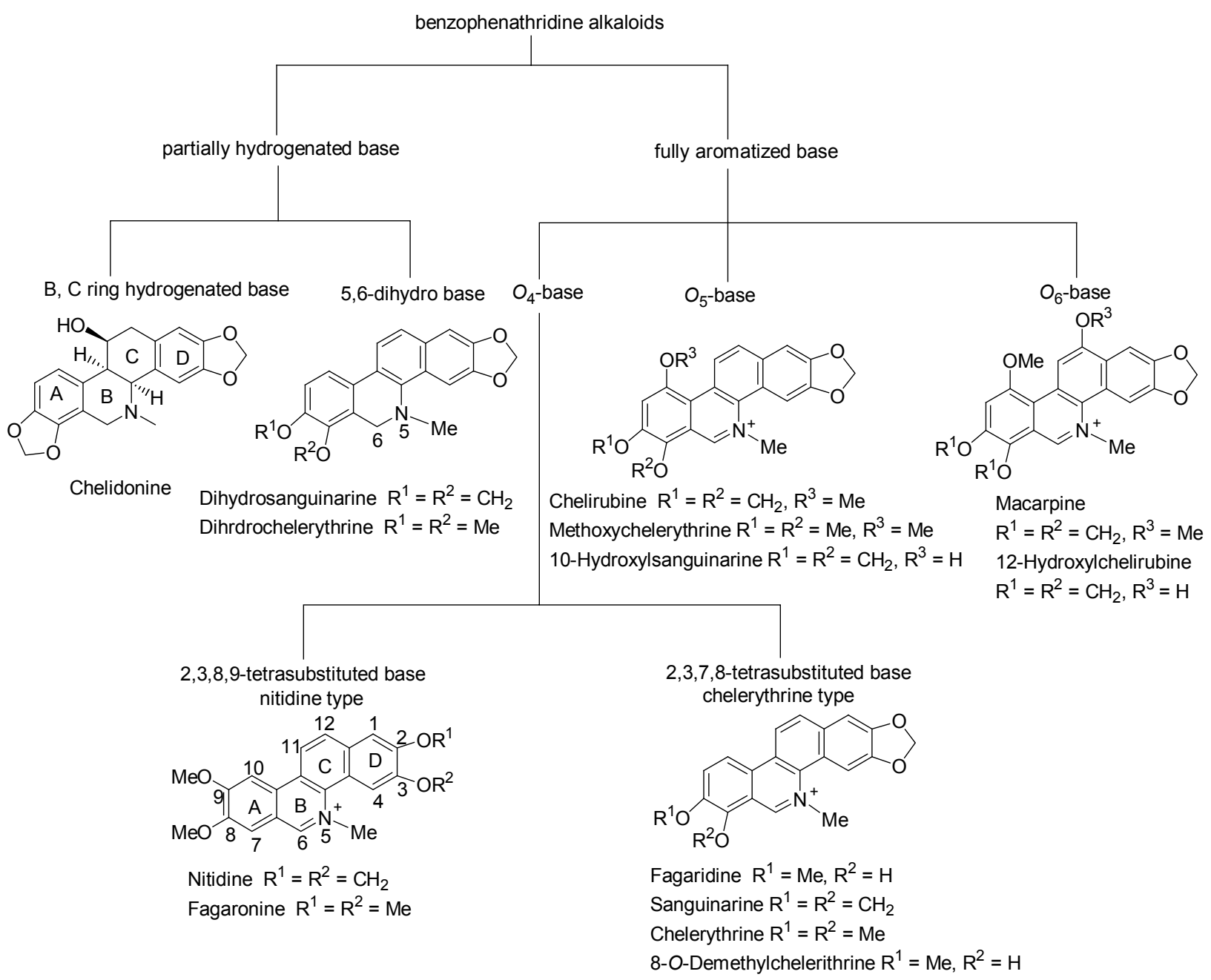

Scheme 2

基或羟基取代个数, 其中 $\mathrm{O}_{4}$-生物碱可以依据母核取代 位置的不同进一步分为两面针碱型(nitidine type)和白屈 菜红碱型两类(chelerythrine type).

虽然苯并啡啶类生物碱的生源合成途径已明确, 但 目前没有相关文献报道 Protopine 6 位高效选择性羟基 化反应, 直接将 Protopine 转化为二氢血根碱的化学合 成仍然存在困难和挑战. 因此, 苯并啡啶类生物碱衍生 物的合成通常采用常规化学原料通过多步合成来实现. 在大部分合成路线中, B 环和 C 环的环合反应是构建苯 并啡啶生物碱四环体系的最后步骤. 本文将依据构建苯 并啡啶基本骨架环合反应的成键位置, 将文献报道的合 成路线进行综述和分类(Scheme 3).

\section{B 环环合策略}

\section{$1.1 \mathrm{C}_{6}-\mathrm{C}_{6 \mathrm{a}}$ 成键环合}

\subsubsection{Bischler-Napieralski 环合反应}

Bischler-Napieralski (BN) 反应是合成异喹啉类生物 碱的常用合成方法之一 ${ }^{[9]}$, 苯并啡啶类生物碱基本骨架

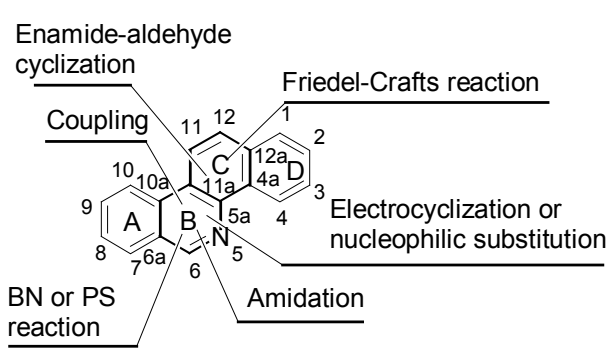

\section{Scheme 3}

中 A-B 环构成异喹啉结构单元. BN 反应构建苯并啡啶 基本骨架一般以 1 -四氢荎酮(1)为关键合成前体 ${ }^{[10]}$, 在 Lewis 酸 $\mathrm{TiCl}_{4}$ 的催化下 1 -四氢萗酮(1)与甲胺反应得到 中间体烯胺，随后用 $\mathrm{NaBH}_{4}$ 还原得到仲胺 2. 中间体 2 氮原子甲酰化后, $\mathrm{DDQ}$ 氧化 $\mathrm{C}$ 环芳构化制得 $\mathrm{BN}$ 反应前 体 4. 三氯氧磷催化中间体 4 发生 $\mathrm{BN}$ 反应, $\mathrm{C}_{6}-\mathrm{C}_{6 \mathrm{a}}$ 成 键环合后得到季铵类苯并啡啶类生物碱基本骨架 $\mathbf{5}$ (Scheme 4). Satio 等 ${ }^{[1]}$ 的研究表明固体光气能够替代三 氯氧磷有效催化 BN 反应顺利进行，收率 78\% 91\%。 
<smiles>[R][R]1ccc2c(c1)C(NC)C(c1ccccc1)CC2</smiles><smiles>[R]c1ccc(C2CCc3cc([R])ccc3C2N(C)C)cc1</smiles>
3<smiles>[R]c1ccc(-c2ccc3ccccc3c2N(C)C=O)cc1</smiles>

Scheme 4

以 1 -四氢萗酮 $(6)$ 为起始原料在合成 $B / C$ 环氢化苯 并啡啶类生物碱方面得到了较好的应用. Yoshida 等 ${ }^{[12]}$ 以 $\mathrm{DMF}$ 为溶剂, 铁粉/ $\mathrm{TMSCl}$ /甲酸/醋酸酐肜体系催化 中间 7 还原氨基化转化为相应的甲酰烯胺中间体 $\mathbf{8}$, 其 氮甲基化产物 $9 \mathrm{BN}$ 环化后，用硼氢化钠还原 5,6 位双键 生成烯胺中间体 $\mathbf{1 0}$. 在强酸性条件下, $\mathrm{NaBH}_{3} \mathrm{CN}$ 还原中 间体 10 得到目标产物 11 (Scheme 5). 上述还原过程具 备立体专一性, 烯胺中间体 $\mathbf{1 0}$ 在酸性条件下转化为季 铵类 Shiff 碱, 氢负试剂从立体位阻较小的一侧亲核加 成 Shiff 碱中间体得到 $\mathrm{B} / \mathrm{C}$ 环顺式产物.

2000 年 Ishikawa 等 ${ }^{[13]}$ 在系统地研究苯并啡啶母核<smiles>COc1cc(C2CCc3cc4c(cc3C2=O)OCO4)cc(OC)c1OC</smiles><smiles>COc1cc(C2=C(NC=O)c3cc4c(cc3CC2)OCO4)cc2c1OCO2</smiles><smiles>COc1cc2c(c(OC)c1OC)CN(C)C1=C2CCc2cc3c(cc21)OCO3</smiles>

Reagents and conditions: (a) $\mathrm{NH}_{2} \mathrm{OH} \cdot \mathrm{HCl}, \mathrm{K}_{2} \mathrm{CO}_{3}, \mathrm{EtOH} .90 \%$; (b) $\mathrm{Fe}$, TMSCl, $\mathrm{HCO}_{2} \mathrm{H}-\mathrm{Ac}_{2} \mathrm{O}$, DMF, $87 \%$; (c) $\mathrm{Me}_{2} \mathrm{SO}_{4}, \mathrm{PhCH}_{2} \mathrm{~N}(\mathrm{Et})_{3} \mathrm{Cl}$, $20 \% \mathrm{NaOH}$ aq., $100 \%$; (d) $\mathrm{POCl}_{3}$ then $\mathrm{NaBH}_{4}, 75 \%$; (e) $\mathrm{NaBH}_{3} \mathrm{CN}$, $\mathrm{MeOH}$, conc. $\mathrm{HCl}, 70 \%$

\section{Scheme 5}

$\mathrm{A}$ 环上烷氧基或羟基取代位置对三氯氧磷催化 $\mathrm{BN}$ 反应 环合产物产率的影响时发现了非常有趣的结果。如 Scheme 6 所示，当 $\mathrm{A}$ 环 10 位有甲氧基或差基取代时，中 间体 12 苯环 $10 \mathrm{a}$ 位碳原子作为亲核体加成 $\mathrm{C}=\mathrm{N}$ 双键, 随后发生分子内重排，导致 $\mathrm{A}$ 环扩环产物的产生，形成 新颖的吡咯类衍生物 13,14 (Scheme 6).

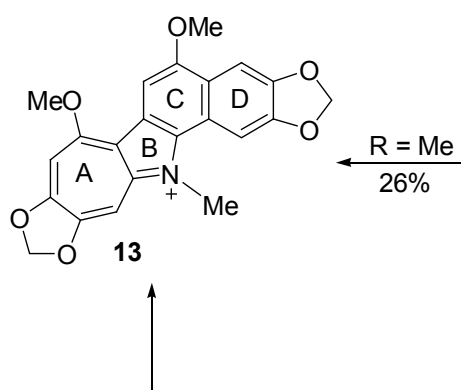

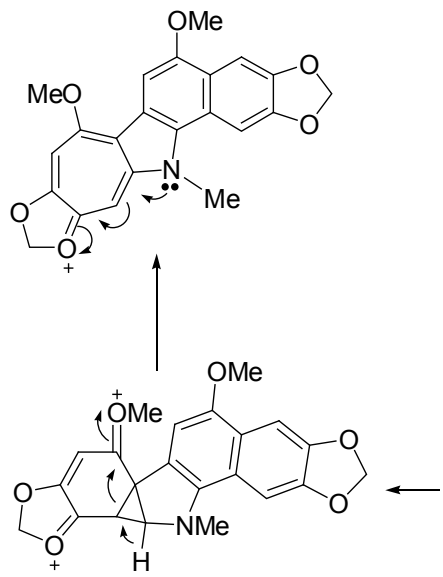<smiles></smiles><smiles>COCCOC(=O)c1cc2c(cc1OC)OCO2</smiles>

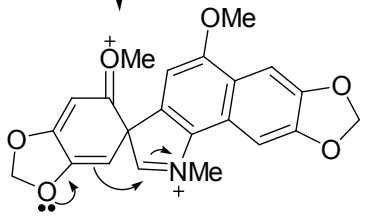

Scheme 6 
$\mathrm{BN}$ 反应原料 $N$-酰基芳香胺中间体 12 一般以 1-四 氢荎酮为原料制备. Treus 等 ${ }^{[14]}$ 报道了一种全新的制备 该中间体的方法用于合成苯并啡啶类生物碱. 该方法以 异喹啉生物碱 15 为原料, 在 LDA 的作用下, 中间体 15 发生 $\mathrm{C}-\mathrm{N}$ 键断裂生成苯乙烯中间体 16. Pd-C 催化中间 体 16 环合制得 $N$-酰基芳香胺 $\mathbf{1 7}$, 随后在三氯化磷和五 氧化磷的作用环合生成苯并啡啶类化合物 18 (Scheme 7).<smiles>[R]c1ccc(/C=C/c2cc(OC)c(OC)cc2/C=C/c2cc(/C=C3\c4cc(OC)c(OC)cc4CCN3C(=O)CC)ccc2[R])cc1[R]</smiles><smiles>[R]c1cc2c(=O)n(C)c3c4cc(OC)c(OC)cc4ccc3c2cc1[R]</smiles>

Reagents and conditions: (a) LDA, THF, $0{ }^{\circ} \mathrm{C}, 30 \sim 60 \mathrm{~min}, 100 \%$; (b) (i) $10 \% \mathrm{Pd}-\mathrm{C}$, o-xylene, reflux, $3 \sim 6 \mathrm{~d}, 35 \% \sim 50 \%$; (ii) $\mathrm{NaH}$, THF, r.t., $30 \mathrm{~min}$, Mel, r.t., $40 \mathrm{~min} \sim 1 \mathrm{~h}, 74 \% \sim 75 \%$; (c) $\mathrm{P}_{2} \mathrm{O}_{5}$, $\mathrm{POCl}_{3}$, reflux, $1.5 \sim 2.5 \mathrm{~h}, 58 \% \sim 66 \%$

\section{Scheme 7}

\subsubsection{Pictet-Spengler (PS) 环合反应}

$\mathrm{PS}$ 反应与 $\mathrm{BN}$ 反应类似, 均为 Manich 反应的变异 类型. Vicario 等 ${ }^{[15]}$ 在 $-78{ }^{\circ} \mathrm{C}$ 下, 用 Lewis 酸 $\mathrm{TiCl}_{4}$ 催化 1-四氢萗酮(19)与苠胺反应生成 Shiff 碱, 随后在 -20 ${ }^{\circ} \mathrm{C}$ 下用 $\mathrm{NaBH}_{4}$ 立体专一性还原 19 得到中间体 20. 酸性 条件下 20 与甲醛发生 $\mathrm{PS}$ 反应 $\mathrm{C}_{6}-\mathrm{C}_{6 \mathrm{a}}$ 成键环合生成 $\mathrm{B} / \mathrm{C}$ 环顺式目标产物 21 (Scheme 8).<smiles>[R]c1cc2c(cc1[R])[C@H](N)[C@H](c1cc([R])c([R])c([R])c1)CC2</smiles><smiles>[R]c1cc2c(cc1[R])[C@@]1(Br)CC[C@H]2c2c(cc([R])c([R])c2[R])C[C@H]1[Z17]</smiles>

Reagents and conditions: (a) (i) $\mathrm{TiCl}_{4}, \mathrm{BnNH}_{2}, \mathrm{Et}_{3} \mathrm{~N}, \mathrm{THF},-78{ }^{\circ} \mathrm{C}$, $70 \% \sim 82 \%$; (ii) $\mathrm{NaBH}_{4}, \mathrm{MeOH},-20{ }^{\circ} \mathrm{C}$. (b) $\mathrm{HCHO}, 1 \mathrm{~mol} / \mathrm{L} \mathrm{HCl}$, $60{ }^{\circ} \mathrm{C}, 86 \% \sim 94 \%$

\section{Scheme 8}

\section{$1.2 \mathrm{~N}_{5}-\mathrm{C}_{6}$ 成键环合}

$\mathrm{N}_{5}-\mathrm{C}_{6}$ 成键环合一般采用分子内 $N$-酰基化反应或 利用氮原子的亲核性质进行分子内 Mitsunobu 反应或亲 核取代反应形成 $\mathrm{C}-\mathrm{N}$ 键，该方法在构建苯并啡啶类生 物碱 B 环体系中并不多见. 2003 年 Watanabe 等 ${ }^{[16]}$ 报道 的一条新颖的合成路线中采用该环合策略. 该小组以苯 乙酰氯 22 为起始原料，与 2-甲基-7-甲氧基苯并呋喃发 生傅克酰基化反应，再与溴乙酸乙酯发生 Reformatsky 反应得到叔醇中间体 23. $\mathrm{Et}_{3} \mathrm{SiH}$ 还原中间体 23 后得到的 产物在三氯氧磷存在下发生分子内傅克反应生成 3-芳 基取代-1-四氢荟酮中间体 24. DDQ 氧化中间体 24 芳构 化后，茮基保护酚差基氧制得关键中间体 25 . 采用硫酸 二甲酯对中间体 25 的氧化开环产物中的羧基和酚羟基 甲基化，随后脱去茮基保护得到中间体 26. 用亚硝基异 戊酯处理中间体 26 制得肟醚中间体 27. $\mathrm{Pd} / \mathrm{C}$ 催化肜醚 中间体 27 还原氨基化, $\mathrm{N}_{5}-\mathrm{C}_{6}$ 成酰胺键得到苯并啡啶 生物碱 28 (Scheme 9). 该合成路线的最大特点是 2-甲基 苯并呋喃母核氧化官能团化和肜醚还原氨基化，同时可 以在单独在苯并啡啶母核 12 位引入官能团酚羟基合成 $\mathrm{O}_{5}$-生物碱.

Mitsunobu 反应是羟基酯化反应的常用方法，在大 环内酯类类天然产物的合成中应用十分广泛 ${ }^{[17]}$. Yoshida 等 ${ }^{[18]}$ 在全合成天然苯并啡啶类生物碱 Homochelidonine (33)的过程中采用了该反应实现 $\mathrm{N}_{5}-\mathrm{C}_{6}$ 成键环合. 在该 合成路线中, 起始原料为具有 1-四氢萗酮结构特征的天 然内酯类化合物 arnottin II (29). $\mathrm{OsO}_{4}$ 氧化 arnottin II 双 键得到邻二醇，随后保护羟基，经羰基肟化、还原氨基 化等步骤得到关键中间体 30, 该中间体㿝化后还原羧 酸羰基再与三氟甲基乙酸䣶反应得到 Mitsunobu 反应原 料 31. 采用 $\mathrm{DEAD} /$ 三苯基膦体系催化 31 完成分子内的 Mitsunobu 反应 $\mathrm{N}_{5}-\mathrm{C}_{6}$ 成键环得到 $\mathrm{B}$ 环环合产物 32 (Scheme 10).

环合反应底物的制备在 $\mathrm{N}_{5}-\mathrm{C}_{6}$ 成键合成苯并啡啶 过程中非常关键. 2008 年 Fleming 等 ${ }^{[19]}$ 利用钯催化氮芐 基保护的氮杂双环烯烃 34 与取代苯基硼酸中间体 $\mathbf{3 5}$ 偶 联反应合成得到中间体 36，脱去甲氧基甲醚保护基后 得到中间体 $\mathbf{3 7}$. 中间体 $\mathbf{3 7}$ 在 Mitsunobu 反应条件下无 法完成 $\mathrm{N}_{5}-\mathrm{C}_{6}$ 成键反应，但在 $\mathrm{CBr}_{4}$ 和三苯基膦存在的 $\mathrm{THF}$ 溶液中 $0{ }^{\circ} \mathrm{C}$ 反应 $1 \mathrm{~h}$ 后可制得苠溴中间体，之后在 碱性条件下完成分子内的亲核取代反应形成 $\mathrm{N}_{5}-\mathrm{C}_{6}$ 单 键得到 B 环环合产物 38 (Scheme 11). 该中间体可用于 全合成天然苯并啡啶类生物碱 homochelidonine (33) 的 合成前体. 反应机理如 Scheme 12 所示, 钯催化合成中 间体 36 的过程开始于硼酸芳香基团过度金属化. 芳基 嗍酸 35 在手型配体 $(S)$-tol-binap 作用下与硼酸化生成芳 
<smiles>CCOC(=O)CC(O)(Cc1cc2c(cc1C(CC)(CC)CCC(=O)Cl)OCO2)c1ccc(OC)c2oc(C)cc12</smiles><smiles>CCOC(=O)CC(Cc1cc2c(cc1C(CC)CC)OCO2)c1ccc(OC)c2oc(C)cc12</smiles><smiles>COc1ccc(-c2cc(OCc3ccccc3)c3cc4c(cc3c2)OCO4)c(C(=O)O)c1O</smiles><smiles>CO/N=C1\c2cc3c(cc2C(=O)CC1c1ccc(OC)c(OC)c1C(=O)OC)C(=O)CO3</smiles>

Reagents and conditions: (a) $\mathrm{SnCl}_{4}, \mathrm{CH}_{2} \mathrm{Cl}_{2},-15{ }^{\circ} \mathrm{C}, 2.5 \mathrm{~h}, 64 \%$; (b) $\mathrm{BrCH}_{2} \mathrm{CO}_{2} \mathrm{Et}, \mathrm{Zn}, \mathrm{l}_{2}$, THF, reflux, $0.5 \mathrm{~h}, 93 \%$; (c) $\mathrm{Et} \mathrm{SiH}_{3} \mathrm{SFA}, \mathrm{CH}_{2} \mathrm{Cl}_{2}, 0$ ${ }^{\circ} \mathrm{C}, 1.5 \mathrm{~h}, 91 \%$; (d) i) $\mathrm{KOH}$ aq., EtOH, reflux, $1 \mathrm{~h}, 100 \%$; ii) $\mathrm{POCl}_{3}, \mathrm{~K}_{2} \mathrm{CO}_{3}, \mathrm{CH}_{3} \mathrm{CN}, 55{ }^{\circ} \mathrm{C}, 3 \mathrm{~h}, 81 \%$; (e) i) $\mathrm{TsOH} \cdot \mathrm{H}_{2} \mathrm{O}$, isopropenyl accetate, 95 ${ }^{\circ} \mathrm{C}, 13 \mathrm{~h}$, ii), DDQ, r.t., $1 \mathrm{~h}$, iii) $\mathrm{NaOH}$ aq, $\mathrm{EtOH}, 85{ }^{\circ} \mathrm{C}, 1 \mathrm{~h}, 89 \%$; (f) $\mathrm{PhCH}_{2} \mathrm{Br}, \mathrm{K}_{2} \mathrm{CO}_{3}$, DMF, $50{ }^{\circ} \mathrm{C}, 2 \mathrm{~h}, 91 \%$; (g) i) OsO $\mathrm{O}_{4}$, pyridine, $30{ }^{\circ} \mathrm{C}, 3 \mathrm{~h}$, ii) $\mathrm{Na}_{2} \mathrm{SO}_{3}$, EtOH, $75{ }^{\circ} \mathrm{C}, 7 \mathrm{~h}, 92 \%$; (h) i) $\mathrm{HIO}_{4}, \mathrm{H}_{2} \mathrm{O}$-dioxane, r.t., 24 h, ii), $\mathrm{NaOH}$ aq., r.t., 4 h, $100 \%$; (i) i) $\mathrm{Me}_{2} \mathrm{SO}_{4}, \mathrm{BnN}^{+} \mathrm{Bu}_{3} \cdot{ }^{\circ} \mathrm{Cl}^{-}, \mathrm{NaOH}^{2}$ aq., benzene, r.t., $3 \mathrm{~h}, 87 \%$, ii) $\mathrm{H}_{2}$, $\mathrm{Pd} / \mathrm{C}$, $\mathrm{AcOH}$, r.t., $3.5 \mathrm{~h}, 82 \%$; (j) i) $i-\mathrm{C}_{5} \mathrm{H}_{11} \mathrm{ONO}, \mathrm{K}_{2} \mathrm{CO}_{3}$, DMF, r.t., 3.5 h, ii) $\mathrm{Me}_{2} \mathrm{SO}_{4}$, r.t., 1.5 h, $86 \%$; (k) $\mathrm{H}_{2}$, $\mathrm{Pd} / \mathrm{C}$ $\mathrm{AcOH}$, r.t., $3.5 \mathrm{~h}, 95 \%$

\section{Scheme 9}

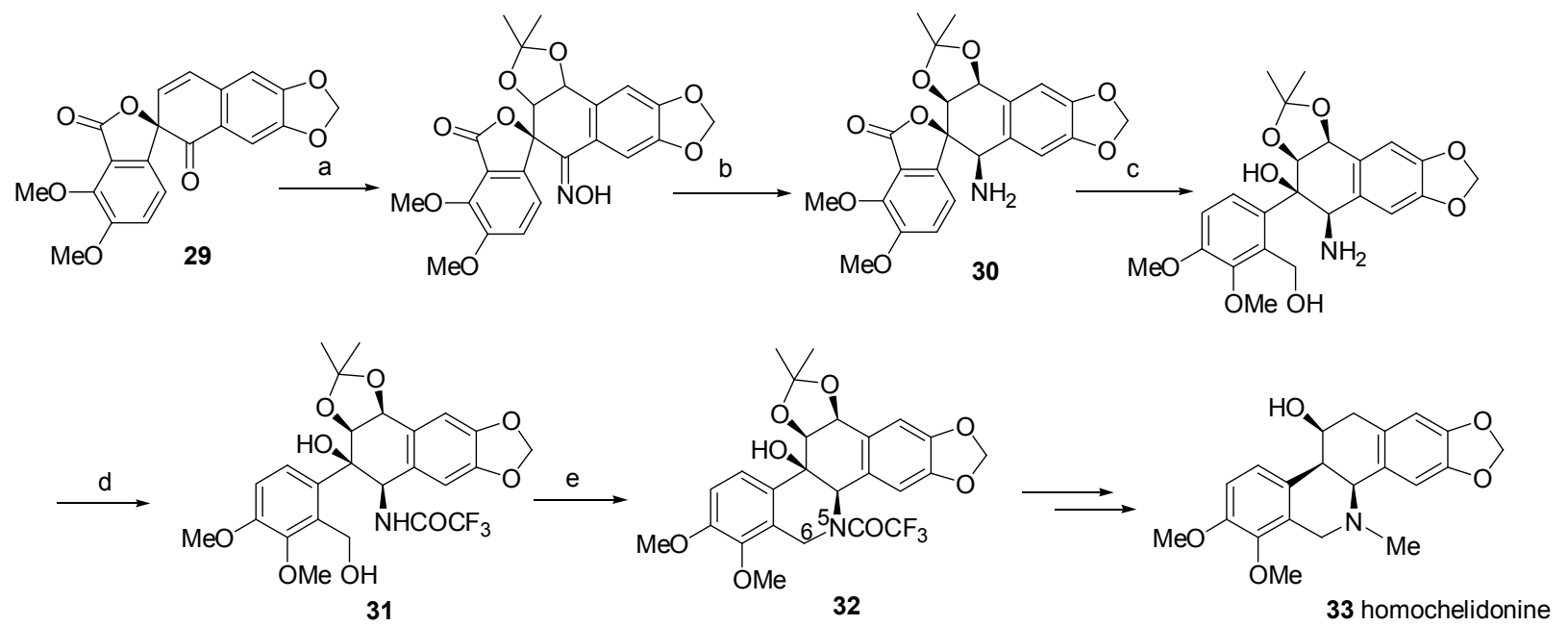

Reagents and conditions: (a) (i) $\mathrm{OsO}_{4}$, pyridine, r.t., 1 h, then $\mathrm{NaHSO}_{3}$ aq., r.t., $20 \mathrm{~h}, 70 \%$ in two steps. (ii) $\left(\mathrm{CH}_{3}\right)_{2} \mathrm{C}\left(\mathrm{OCH}_{3}\right)_{2}, p-\mathrm{TsOH} \cdot \mathrm{H}_{2} \mathrm{O}$, DMF, $90{ }^{\circ} \mathrm{C}, 1 \mathrm{~h}, 98 \%$. (iii) $\mathrm{NH}_{2} \mathrm{OH} \cdot \mathrm{HCl}$, pyridine, $100{ }^{\circ} \mathrm{C}, 6 \mathrm{~h}, 83 \%$; (b) $\mathrm{TiCl}_{3}$ aq., $\mathrm{NaBH}_{3} \mathrm{CN}, \mathrm{NH}_{4} \mathrm{OAc}$, dioxane, r.t., 5 h, $96 \%$; (c) $\mathrm{LiAlH}{ }_{4}$, $\mathrm{THF}$, r.t., 1 h, $95 \%$; (d) $\left(\mathrm{CF}_{3} \mathrm{CO}\right)_{2} \mathrm{O}$, pyridine, $0{ }^{\circ} \mathrm{C}, 2$ h; then $\mathrm{NaHCO}_{3}$ aq., $\mathrm{MeOH}$, r.t., 16 h, $75 \%$ in two steps; (e) $\mathrm{DEAD}, \mathrm{Ph} 3 \mathrm{P}, \mathrm{THF}, \mathrm{r}$. .., 3 h, $83 \%$

Scheme 10 
<smiles>O=[N+]1C2C=CC(c3cc4c(cc32)OCO4)[N+]1([O-])[O-]</smiles>

34

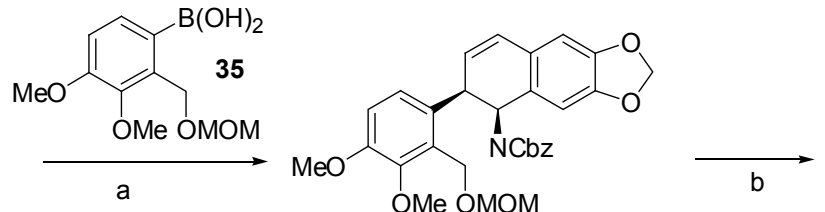

36<smiles>COc1ccc(C2C=Cc3cc4c(cc3C2NC(=O)O)OCO4)c(CO)c1OC</smiles>

37<smiles></smiles>

38

Reagents and conditions: (a) $\left[\mathrm{Pd}(\mathrm{MeCN})_{2} \mathrm{Cl}_{2}\right](5 \mathrm{~mol} \%)$, (S)-tol-binap $(5.5 \mathrm{~mol} \%), \mathrm{Cs}_{2} \mathrm{CO}_{3}, \mathrm{MeOH}, \mathrm{r.t.}, 6 \mathrm{~h}, 80 \%$; (b) $\mathrm{HCl}$, $i-\mathrm{PrOH} / \mathrm{THF}$, r.t., $8 \mathrm{~h}$, $75 \%$; (c) $\mathrm{CBr}_{4}, \mathrm{PPh}_{3}, \mathrm{CH}_{2} \mathrm{Cl}_{2}, 80{ }^{\circ} \mathrm{C}, 1 \mathrm{~h}$, then $\mathrm{NaH}, \mathrm{DMF}, 0{ }^{\circ} \mathrm{C}, 3 \mathrm{~h}, 90 \%$ in two steps

Scheme 11

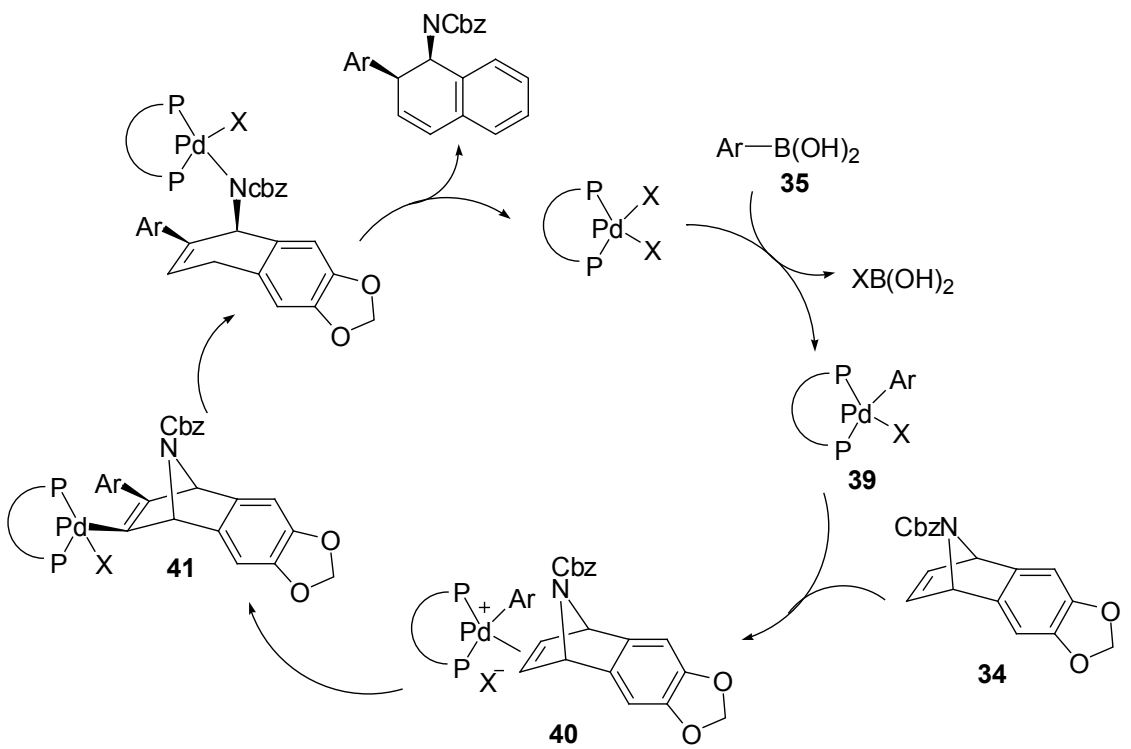

Scheme 12

香 $\mathrm{Pd}^{\mathrm{II}}$ 物种 39, 39 从 $N$-杂双环烯烃(34)立体位阻较小的 一侧与中间体 34 结合生成正离子复合物 40. 该离子复 合物发生顺式碳钯化得到中间体 41, 接着进行 $\beta$-杂原 子消除和解离得到开环化合物 36 和活性 $\mathrm{Pd}^{\mathrm{II}}$ 进入下一 催化循环 ${ }^{[20]}$.

\section{$1.3 \mathrm{~N}_{5}-\mathrm{C}_{5 \mathrm{a}}$ 成键环合}

$\mathrm{N}_{5}-\mathrm{C}_{5 \mathrm{a}}$ 成键策略在苯并啡啶类生物碱母核合成中 较为少见. 2011 年 Ishihara 等 ${ }^{[21]}$ 利用微波辅助电环化反 应 $\mathrm{N}_{5}-\mathrm{C}_{5 \mathrm{a}}$ 成键合成苯并啡啶生物碱 A-D 环基本骨架. 在该合成路线中, 起源原料为邻溴苯甲醛(42)和邻二醇 硣酸酯(43). 42 和 43 在钯催化下发生 Suzuki-Miyaura 偶 联反应得到另一苯甲醛中间体 $\mathbf{4 4}$. 中间体 44 与 $O$-甲基 羟胺反应得到肜醚 45 . 在 $180{ }^{\circ} \mathrm{C}$ 微波辐射条件下, 中间 体 45 发生分子内电环化反应得到 11,12-二氢苯并啡啶
(46)，随后用 Pd-C 催化中间体 46 脱氢芳构化得到 A-D 环全芳香化目标产物 47 (Scheme 13). 上述合成路线的 缺点在于邻二醇硣酸酯原料 43 合成路线步骤较多, 影 响了总收率 ${ }^{[22]}$.

$\mathrm{N}_{5}-\mathrm{C}_{5 \mathrm{a}}$ 成键过程也可利用一些特殊苯并啡啶类化 合物 $\mathrm{C}$ 环 $5 \mathrm{a}$ 位碳原子缺电子特性和 5 位氮原子的亲核 性质进行分子内的芳香亲核取代反应来完成 B 环合过 程. Lysén 等 ${ }^{[23]}$ 以 1-氟慕(48)为原料，在 2,2,6,6-四甲基哌 啶锂(LiTMP)的作用下发生选择性邻位芳香碳去质子 化，高亲核性碳负离子与异丙基硼酸酯反应得到对应的 1-氟取代硼酸酯中间体 49. 该中间体与邻碘苯氰 $(50)$ 在 钯催化下经 Suzuki-Miyaura 偶联制得 1-氟代-2(2'-氭基 苯基)䒬(51). 苯环上氧基被正丁基锂加成后生成的氮负 离子亲核取代荟环 1 位氟原子, $\mathrm{N}_{5}-\mathrm{C}_{5 \mathrm{a}}$ 成键即可得到全 
<smiles>CC(C)COc1cc2c(cc1C1=Cc3cc4c(cc3OCO1)OCC4)OCO2</smiles><smiles>CC(C)(C)C1COc2cc3c(cc21)CCc1c-3ncc2cc3c(cc12)OCO3</smiles>

Reagents and conditions: (a) $\mathrm{PdCl}_{2}\left(\mathrm{PPh}_{3}\right)_{2}, \mathrm{~K}_{2} \mathrm{CO}_{3}, \mathrm{MeOH}$, DMF, $80{ }^{\circ} \mathrm{C}, 0.5 \sim 1 \mathrm{~h}, 97 \%$; (b) $\mathrm{MeONH}_{2} \cdot \mathrm{HCl}, \mathrm{AcONa}, \mathrm{EtOH}, 80{ }^{\circ} \mathrm{C}, 1 \mathrm{~h}, 98 \%$; (c) microwave, 1,2-dichlorobenzene, $180{ }^{\circ} \mathrm{C}, 94 \%$; (d) $10 \% \mathrm{Pd}-\mathrm{C}, 1$,2-dichlorobenzene, $180{ }^{\circ} \mathrm{C}, 74 \%$

\section{Scheme 13}

芳香化苯并啡啶母核 52 (Scheme 14), 上述合成路线最 大的优点是合成步骤大幅缩短, 而且每步转化率都很 高, 极大地提高了苯并啡啶母核合成效率. 但是该方法 必须采用缺电子的 1-氟菜为原料, 当萗环上有供电子基 团取代时, 很难实现 $\mathrm{N}_{5}-\mathrm{C}_{5 \mathrm{a}}$ 成键.

\section{$1.4 \mathrm{C}_{10 \mathrm{a}}-\mathrm{C}_{11 \mathrm{a}}$ 成键环合}

苯并啡啶基本骨架 $\mathrm{B}$ 环 $\mathrm{C}_{10 \mathrm{a}}-\mathrm{C}_{11 \mathrm{a}}$ 成键环合反应是 近年来这类生物碱合成方法学研究的热点, 主要成键反 应类型为分子内芳基-芳基偶联反应，其中钯催化芳基 偶联反应是最近研究较多的一个反应类型. 该方法具有 合成步骤少、偶联反应转化率高等优点, 在合成苯并啡 啶基本骨架中的应用较为广泛 ${ }^{[24 ~ 27]}$. 钯催化偶联合成
路线中一般采用邻取代苯甲酸 $(\mathbf{5 3})$ 和取代 1-萗胺衍生物 55 为原料. 以取代苯甲酸 53 为原料制备得到对应的酰 氯中间体 54, 该中间体与 55 反应得到酰胺 56 . 醋酸钯 作为催化剂可顺利催化酰胺 56 分子内的芳基偶联反应, $\mathrm{C}_{10 \mathrm{a}}-\mathrm{C}_{11 \mathrm{a}}$ 成键环合生成 6-O-苯并啡啶(57). $\mathrm{LiAlH}_{4}$ 还原 57 酰胺羰基生成的 6-着基烡并啡啶在酸性条件下脱水 得到季铵类苯并啡啶生物碱 58 (Scheme 15).

Harayama 等 ${ }^{[28]}$ 在合成白屈菜红碱的过程中发现, $\mathrm{PPh}_{3}, \mathrm{P}(o-\mathrm{Tol})_{3}, \mathrm{Bu}_{3} \mathrm{P}, \mathrm{DPPP}$ 等不同膦配体和 $\mathrm{Ag}_{2} \mathrm{CO}_{3}$ 和 响到分子内偶联反应的区域选择性. 当采用 $\mathrm{PPh}_{3} / \mathrm{Ag}_{2} \mathrm{CO}_{3}$, $\mathrm{Na}_{2} \mathrm{CO}_{3}$ 等缚酸剂组合均可得催化反应发生得到较高产 率的偶联产物, 但是膦配体和缚酸剂的选择可以影响到<smiles>CCCCc1nc2c3ccccc3ccc2c2ccc(C(C)(C)C)cc12</smiles>

Scheme 14
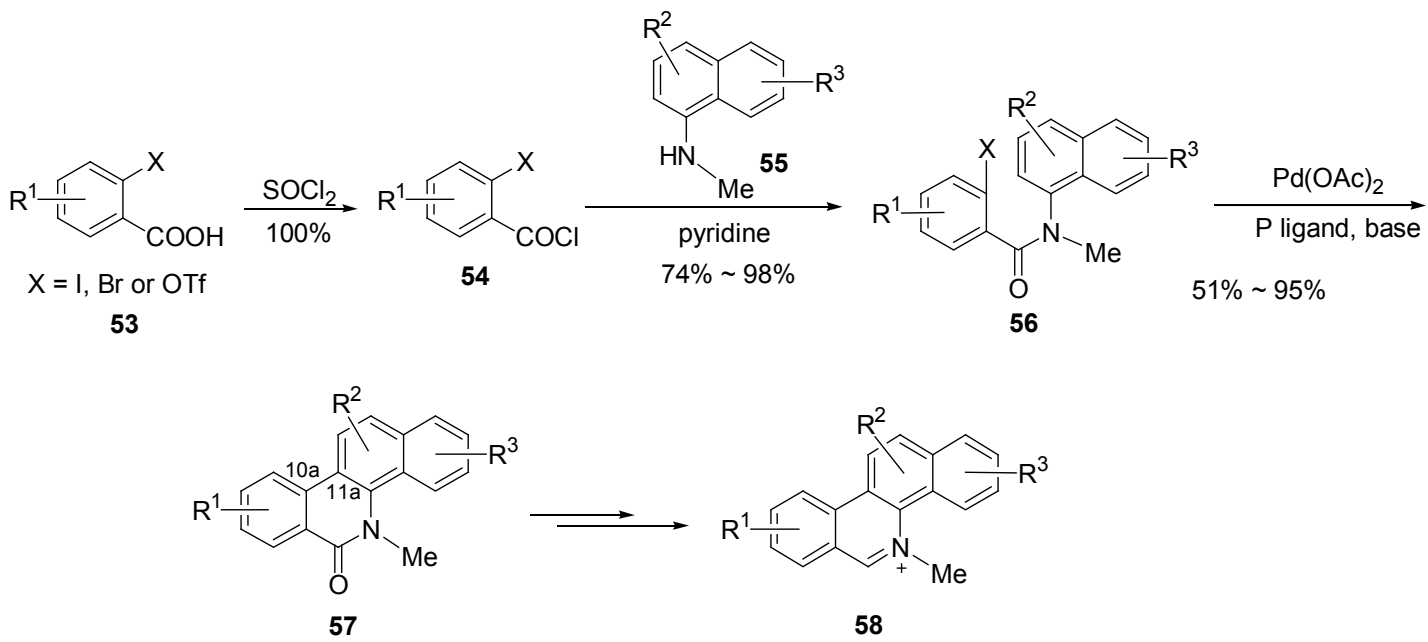

Scheme 15 
分子内偶联反应的区域选择性. 当采用 $\mathrm{P}(o-\mathrm{Tol})_{3} /$ $\mathrm{Na}_{2} \mathrm{CO}_{3}$ 或 $\mathrm{PPh}_{3} / \mathrm{Na}_{2} \mathrm{CO}_{3}$ 体系时, 将会有 $16 \% \sim 20 \%$ 的 $\mathrm{C}_{10 \mathrm{a}}-\mathrm{C}_{4}$ 偶联产物 61 的产生; 而采用 $\mathrm{P}(o-\mathrm{Tol})_{3} / \mathrm{Ag}_{2} \mathrm{CO}_{3}$ 体系时, 仅有 $1 \%$ 的 $\mathrm{C}_{10 \mathrm{a}}-\mathrm{C}_{4}$ 偶联产物生成, $\mathrm{C}_{10 \mathrm{a}}-\mathrm{C}_{11 \mathrm{a}}$ 偶联目标产物 60 的收率可达 96\% (Eq. 1).<smiles>COc1ccc(I)c(C(=O)N([O-])c2cccc3cc4c(cc23)OCO4)c1OC</smiles>

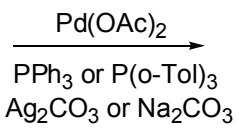

59

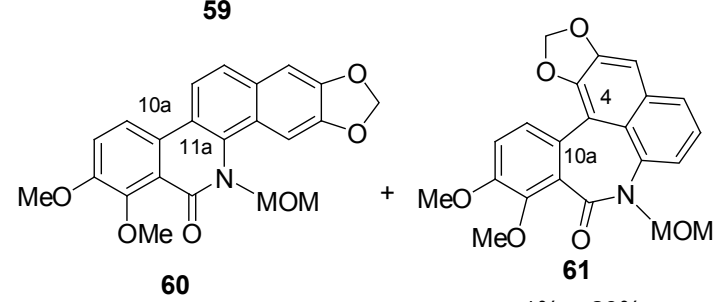

$1 \% \sim 20 \%$

$51 \% \sim 96 \%$

分子内自由基偶联反应是 $\mathrm{B}$ 环 $\mathrm{C}_{10 \mathrm{a}}-\mathrm{C}_{11 \mathrm{a}}$ 成键环合的 另一有效途径, 反应所需原料与钯催化偶联反应类 似 ${ }^{[29]}$. Nakanishi 等 ${ }^{[30]}$ 在合成具有抗癌活性的季铵类苯 并啡啶生物碱 NK109 的过程中, 以邻溴代苯甲醛(62)和
取代 1-菜胺 $(63)$ 为原料反应制备得到苯胺衍生物 64. 在 自由基引发剂 $n-\mathrm{Oct}_{3} \mathrm{SnH}$ 和 $\mathrm{AMBN}$ 作用下 64 中 $\mathrm{C}_{10 \mathrm{a}}-$ $\mathrm{C}_{11 \mathrm{a}}$ 偶联得到 5,6 二氢苯并啡啶, 随后经 $\mathrm{MnO}_{2}$ 氧化得到 中间体 65, 中间体 65 经 $N$-甲基化、脱去苠基保护后得 到 NK109 (66) (Scheme 16). 上述合成路线与钯催化偶 联反应相比，虽然目标产物收率降低，但是反应底物 (64)中游离的氨基对自由基偶联反应没有影响, 无需对 5 位氮原子进行繁琐的保护-脱保护处理. Nakanishi 等 ${ }^{[31}$ 还发现在一 $78{ }^{\circ} \mathrm{C}$ 下用强碱 LDA 处理中间体 64 后 快速升温同样可以得到较低收率的 $\mathrm{C}_{10 \mathrm{a}}-\mathrm{C}_{11 \mathrm{a}}$ 偶联产物, 并有大量未知副产物生成. 该反应的机理可能与形成活 性苯炔中间体相关.

2007 年 Budén 等 ${ }^{[32]}$ 发现光照可以代替 $n-$ Oct $_{3} \mathrm{SnH} /$ $\mathrm{AMBN}$ 体系引发剂自由基偶联反应. 在以录灯作为激 发光源的条件下, $N$-(2-氯苯基)-1-菜胺(67)在液氨中用 $t$ - $\mathrm{BuOK}$ 处理反应 $3 \mathrm{~h}$ 可以实现 $\mathrm{C}_{10 \mathrm{a}}-\mathrm{C}_{11 \mathrm{a}}$ 偶联得到全芳 香化苯并啡啶基本骨架 68 (Scheme 17). 该反应为典型 自由基亲核取代 $\left(\mathrm{S}_{\mathrm{RN}} 1\right)$ 机理，目标产物收率可以达到 91\%. 但是反应底物 67 中荟环和苯环上其它取代基的 存在对偶联反应的转化率是否存在影响, 目前还没有相 关文献报道.<smiles>COc1ccc(Br)c(CNc2cccc3cc4c(cc23)OCO4)c1CNc1ccc(OC)c(OCC(C)C)c1OCC(C)C</smiles>

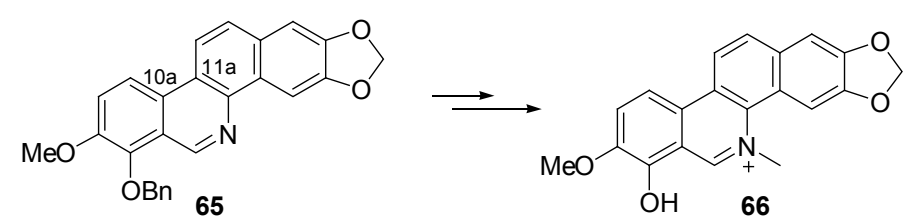

Reagents and conditions: (a) toluene, reflux, $1.5 \mathrm{~h}$; then $\mathrm{Me}_{2} \mathrm{NHBH}_{3}, \mathrm{AcOH}$, toluene, $20{ }^{\circ} \mathrm{C}, 1 \mathrm{~h}, 75 \%$ in two steps; (b) $n$-Oct ${ }_{3} \mathrm{SnH}, \mathrm{AMBN}$, toluene, $110{ }^{\circ} \mathrm{C}, 70 \mathrm{~min}$; then $\mathrm{MnO}_{2}$, toluene, r.t., $1 \mathrm{~h}, 57 \%$ in two steps

\section{Scheme 16}<smiles></smiles>

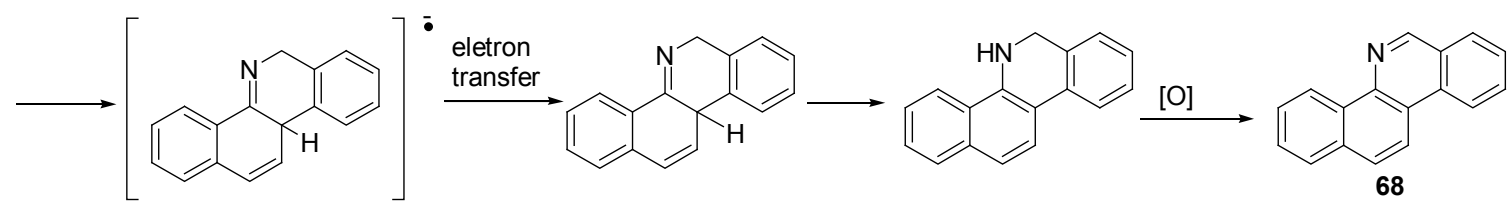

Scheme 17 
在大多数 $\mathrm{C}_{10 \mathrm{a}}-\mathrm{C}_{11 \mathrm{a}}$ 偶联反应成键环合策略中, 苯 甲醛或苯甲酸作为反应原料, 其羰基邻位芳香碳必须进 行卤代官能团化, 这样增加了合成步骤影响目标产物的 最终得率. Moreno 等 ${ }^{[33]}$ 报道了一种利用高价碘试剂 PIFA (69)引发的自由基偶联反应. 在二氯甲烷中, 底物 酰胺 70 在 PIFA 作用下, 反应 $14 \mathrm{~h}$ 后 $\mathrm{C}_{10 \mathrm{a}}-\mathrm{C}_{11 \mathrm{a}}$ 成键生 成 B 环环合产物 71 (Eq. 2), 得率为 40\%. 该自由基反应 无需采用邻位卤代苯甲酸作为原料, 有效地缩短了合成 路线.

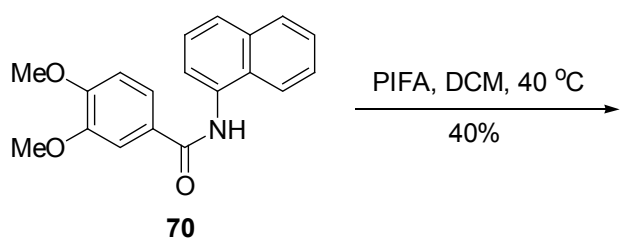<smiles>COc1cc2c(=O)[nH]c3c4ccccc4ccc3c2cc1OC</smiles>

69

\section{5 其它 B 环环合方法}

\subsection{1 钯催化两组份串联反应 B 环环合}

$\mathrm{C}_{10 \mathrm{a}}-\mathrm{C}_{11 \mathrm{a}}$ 钯催化偶联 $\mathrm{B}$ 环环合方法一般采用分子内 芳基-芳基偶联策略，在合成苯并啡啶生物碱基本骨架 研究方面发挥了重要的作用. 在此基础上, 科学家们对 钯催化 B 环成环方法进行了更深入的研究, 其中以钯催 化两组份串联反应构建苯并啡啶基本骨架 $\mathrm{B}$ 环是最近 报道的较多的一个反应类型. 2006 年 Konno 等 ${ }^{\left[{ }^{[4]}\right.}$ 以邻溴 苯甲酸叔丁酯 $(72)$ 和四氢萗酮(73)为原料, 在钯催化下
发生两组份偶联反应生成内酯 74, 该化合物可以作为 天然内酯类化合物 arnottin II (29)的合成前体. 2011 年 Ito 等 ${ }^{[35]}$ 以 74 为原料, 用甲胺处理后得到内酰胺衍生物 75. 中间体 75 经氧化芳构化、羰基还原、脱水后可以得 到白屈菜红碱(Chelerythrine) (Scheme 18).

2011 年, $\mathrm{Lv}$ 等 ${ }^{[36]}$ 以 $N$-杂双环烯烃 $(76)$ 和邻碘苯甲酸 甲酯(77)为原料, 钯催化两组分串联反应直接构建苯并 啡啶基本骨架，该方法可用于血根碱的合成并且总收率 较高.上述合成路线与 2008 年 Fleming 等报道的 homochelidonine (33)合成路线存在类似之处, 主要改进 在于以邻碘苯甲酸甲酯(77)为原料与氮杂双环烯烃(76) 反应(Scheme 19). 串联反应可以一步实现 $\mathrm{N}_{5}-\mathrm{C}_{6}$ 和 $\mathrm{C}_{10 \mathrm{a}}-\mathrm{C}_{11 \mathrm{a}}$ 成键构建 $\mathrm{B}$ 环, 但该反应过程是否具备立体 专一性，目前还未见报道. 镍催化剂 $\mathrm{NiBr}_{2}$ (dppe)可以替 代钯催化类似的串联反应，但是当 $\mathrm{A}$ 或 $\mathrm{D}$ 环上有供电子 烷氧基或羟基存在时, $\mathrm{NiBr}_{2}$ (dppe)不能催化反应进 行 ${ }^{[37,38]}$.

2011 年 Blanchot 等 ${ }^{[39]}$ 发展了一类新颖的钯催化两 组份串联芳基化/氮-芳基化方法用于合成苯并啡啶基本 骨架. 该方法以 1-荎酚三氟甲基磺酸酯(79)和 $N$-硅基醛 亚胺 $(80)$ 为反应原料, 在 $\mathrm{Pd}(\mathrm{OAc})_{2} / \mathrm{PPh}_{3} / \mathrm{Cs}_{2} \mathrm{CO}_{3} /$ norbornene 体系催化下 $\mathrm{N}_{5}-\mathrm{C}_{5 \mathrm{a}}$ 和 $\mathrm{C}_{10 \mathrm{a}}-\mathrm{C}_{11 \mathrm{a}}$ 成键得到苯并啡啶生 物碱 81, 得率达到 $88 \%$ (Eq. 3). Candito 等 ${ }^{[40]}$ 对钯催化机 理进行了探讨, 零价钯催化剂和 1-菜酚三氟甲基磺酸酯 (79)在缚酸剂 $\mathrm{Cs}_{2} \mathrm{CO}_{2}$ 作用下与 norbornene 发生碳钯化产 生芳基钯中间体 82, 接着与 $N$-硅基醛亚胺反应得到 $\mathrm{Pd}(\mathrm{IV})$ 中间体 83, 随后经化学选择性还原消除和去碳钯 化后得到中间体 84. 中间体 84 转化为 85 后经还原消除 得到目标产物 81. 上述合成路线所用原料易得, 若以 $N$ 硅基酮亚胺代替 80 , 在相同条件下反应仍然能够顺利 进行, 并能够在目标产物 81 的 6 位直接引入烷基取代 基，极大的提高了苯并啡啶类衍生物的合成效率 (Scheme 20).<smiles>COc1ccc(Br)c(C(=O)OCc2ccccc2)c1OC</smiles>

72

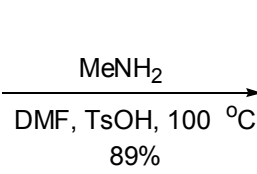

$89 \%$<smiles>O=C1CCCc2cc3c(cc21)OCO3</smiles>

73

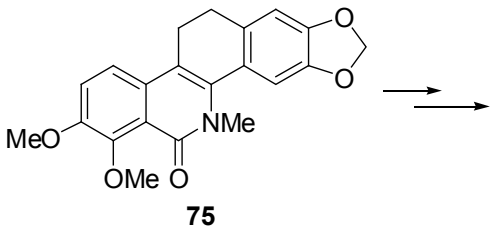

$\underset{\text { toluene, } 15 \mathrm{~h}, 80^{\circ} \mathrm{C}}{\stackrel{\mathrm{Pd}_{2}(\mathrm{dba})_{3}, \mathrm{~K}_{3} \mathrm{PO}_{4}}{\longrightarrow}}$ $78 \%$

$\mathrm{MeO}$

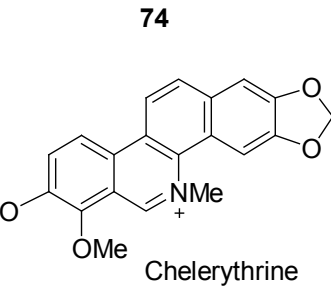

Scheme 18 
<smiles>O=C1NC2C=CC1c1cc3c(cc12)OCO3</smiles>

76

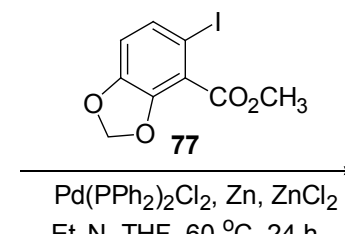

$\mathrm{Et}_{3} \mathrm{~N}, \mathrm{THF}, 60{ }^{\circ} \mathrm{C}, 24 \mathrm{~h}$

$91 \%$

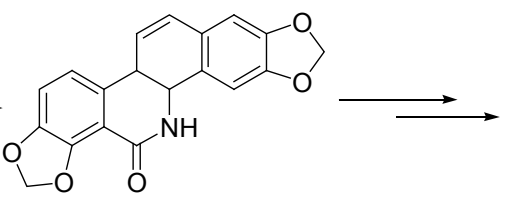

78<smiles>C[n+]1cc2c3c(ccc2c2ccc4cc5c(cc4c21)OCO5)OCO3</smiles>

sanguinarine

Scheme 19<smiles>CCCc1cccc2cc3c(cc12)OCO3</smiles>

79

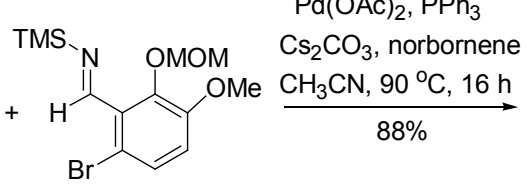

80<smiles>COc1ccc2c(c1)C=NC1c3cc4c(cc3C=C[C@@H]21)OCO4</smiles>

(3)
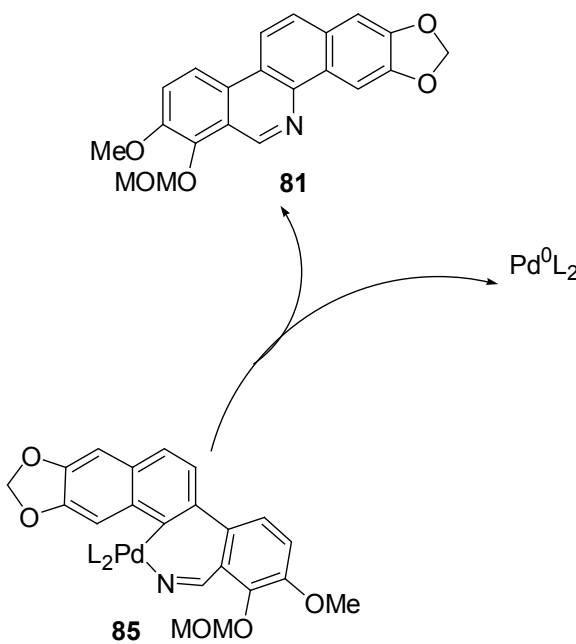

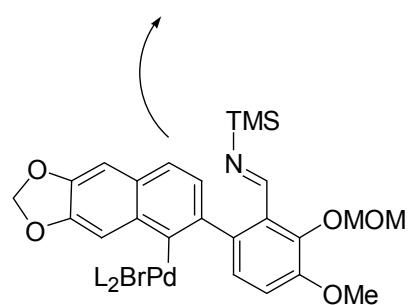

84<smiles>CCCCC1C=CC2CCC12</smiles>

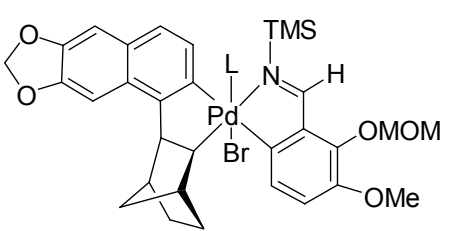

83

Scheme 20

\section{C 环环合策略}

\section{1 分子内烯酰胺-醛环化反应 $\mathrm{C}_{11}-\mathrm{C}_{11 \mathrm{a}}$ 成键环合}

Cho 课题组 ${ }^{[41]}$ 发展了一类新颖的分子内烯酰胺-醛 环化反应用于构建苯并啡定类生物碱基本骨架．在这一 合成路线中，邻甲基苯甲酰胺 $(\mathbf{8 6})$ 在正丁基锂的作用下 甲基去质子化得到碳负离子. 该碳负离子亲核加成苯甲 氧(87)中的氰基，生成的氮负离子活性中间体与酰胺羰 基反应得到异喹啉中间体 $\mathbf{8 8}$. 中间体 $\mathbf{8 8}$ 中酰胺 $N$-甲基 化后脱去 $\mathrm{MOM}$ 保护基，随后氧化得到苯甲醛中间体 90. 该中间体进一步与磷叶立德试剂反应后制得烯醇甲<smiles>[Ga]Oc1cccc2cc3c(cc12)OCO3</smiles>

79

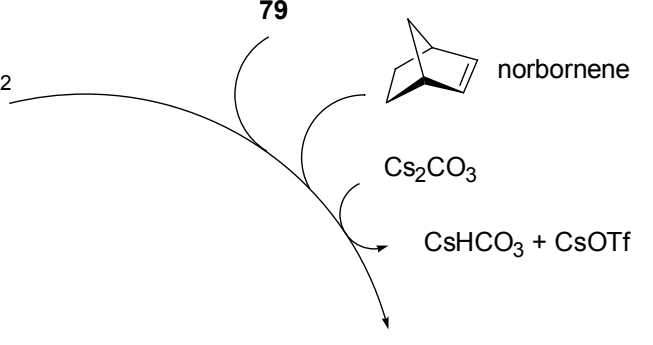<smiles></smiles><smiles>COc1ccc(Br)c(/C=N/N=C/C(C)C)c1OC</smiles>

80 
醚中间体 91. 在酸性条件下 91 发生分子内烯酰胺-醛环 化反应, $\mathrm{C}_{11}-\mathrm{C}_{11 a}$ 成键得到 6- $O$-苯并啡啶(93), 目标产

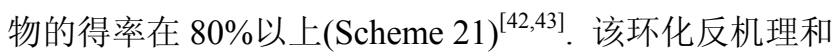
Manich 反应类似，同时与苯并啡啶生源合成途径中的 自发反应过程存在相似之处. 环化反应的底物烯醇甲醚 中间体 91 可以直接用苯乙醇(94)代替 ${ }^{[44,45]}$. 在沙瑞特试 剂 PCC 和醋酸钠的作用下中间体 94 转化为目标产物 $\mathbf{9 3}$, 得率 $60 \%$ 左右(Eq. 4). 若将苯乙醇中间体 94 转化为苯乙 酸乙酯后, 环合反应可在苯并啡啶母核 11 位引入羟基 得到 $O_{5}$-生物碱 $[6,7]$.

\section{2 分子内傅克反应 $\mathrm{C}_{12}-\mathrm{C}_{12 \mathrm{a}}$ 成键环合}

分子内傅克反应是 $\mathrm{C}$ 环环合构建苯并啡啶类生物 碱基本骨架的常用方法之一 ${ }^{[46]}$. 其中傅克酰基化反应 能够在苯并啡啶母核 12 位单独引入羟基得到 $O_{5}$-生物 碱, 在合成非天然苯并啡啶生物碱衍生物中具有独特的 优势. Lynch 等 ${ }^{[47,48]}$ 在合成具有人 DNA 拓扑异构酶 I 抑 制活性和抗病毒活性的苯并啡啶生物碱过程中, 采用用 了该环合策略. 以苯甲胺 $(\mathbf{9 5})$ 和苯甲醛 $(\mathbf{9 6})$ 为原料, 反 应脱水得到相应的 Shiff 碱, 随后被氧基负离子加成双 键得到中间体 97. 该中间体在 HF 作用下发生分子内傅 克反应、水解得到 3-芳基-4-羊崖基异喹啉中间体 99. 在金 属 $\mathrm{Zn}$ 的作用下中间体 99 与溴乙酸乙酯发生 Reformatski 反应得到 $\beta$-麦基基羒酸酯(100), 随后在酸性条件下经傅 克酰基化反应 $\mathrm{C}_{12}-\mathrm{C}_{12 \mathrm{a}}$ 成键环合制得 $O_{5}$-生物碱 12-羟 基苯并啡啶(101). 此外, 也可使用乙烯基格式试剂加成 中间体 99 制备得到中间体 102, 然后在酸性条件下发生 傅克烷基化反应生成 12 位无取代基目标产物 103
(Scheme 22) ${ }^{[49]}$. 该合成路线中起始原料均可由廉价易 得的苯甲醛衍生物制备得到，因此分子内傅克反应 $\mathrm{C}_{12}-\mathrm{C}_{12 \mathrm{a}}$ 成键环合在苯并啡啶生物碱合成中得到了广 泛的应用。

2006 年 Luo 等 ${ }^{[50]}$ 在制备 $N$-去甲基两面针碱 110 过 程中利用分子内傅克反应实现 $\mathrm{C}_{12}-\mathrm{C}_{12 \mathrm{a}}$ 成键环合, 该 合成路线新颖之处在于环合反应底物芳基乙醛中间体 109 的制备. Luo 等以邻溴代苯甲醛(104)为原料与叔丁 胺反应得到 Shiff 碱中间体 105, 以溴苯衍生物 106 为原 料与 3-炔丁醇偶联反应生成中间体 107. 中间体 105 和 107 在镍催化下环合得到 3-芳基-4-羊美乙基异喹啉中间体 108. 中间体 108 经 Swern 氧化后生成的芳基乙醛中间体 109, 随后在酸性条件下 $\mathrm{C}_{12}-\mathrm{C}_{12 \mathrm{a}}$ 成键环合制得目标产 物 $\mathbf{1 1 0}$ (Scheme 23). 当中间体 $\mathbf{1 0 5}$ 中的溴原子被碘取代 后，醋酸钯也能够催化环合反应进行得到 108 , 但是目 标产物收率只有 $38 \%$. 该合成策略具有反应起始原料易 得, 步骤少, 转化率较高等特点, 在制备环合底物 109 过程中无需使用对水敏感的丁基锂或格氏试剂，避免了 氢化钾等剧毒试剂的使用，有望在合成苯并啡啶生物碱 衍生中得到更加广泛的应用.

\section{3 重排或多组分反应直接构建苯并啡啶多环体 系}

在构建苯并啡啶基本骨架过程中，无论是 $\mathrm{B}$ 环合还 是 $\mathrm{C}$ 环环合策略，其环合反应底物往往需要通过多步合 成制备. 1999 年, Hergueta 等 ${ }^{[51]}$ 报道了一类新颖的重排 反应用于直接构建苯并啡啶生物碱的基本骨架, 整个合

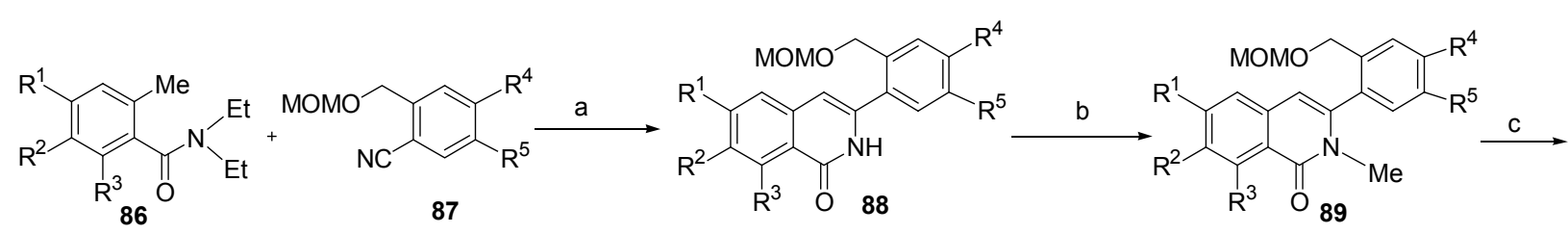<smiles>[R]c1cc2cc([R])c(=O)n(C)c2c([R])c1[R]</smiles>

Reagents and conditions: (a) $n$-BuLi, THF, $50 \% \sim 82 \%$; (b) NaH, Mel, THF, $77 \% \sim 90 \%$; (c) $\mathrm{HCl} 10 \%$; then PDC, DCM, $70 \% \sim 85 \%$; (d) $\mathrm{Ph}_{3} \mathrm{P}^{+} \mathrm{CH}_{2} \mathrm{OMel}^{\prime}, n$-BuLi, THF, $66 \% \sim 74 \%$; (e) $\mathrm{HCl}, 10 \%, 85 \% \sim 88 \%$

\section{Scheme 21}<smiles>[R]c1cc(CCO)c(-c2cc3cc([R])c([R])c([R])c3c(=O)n2C)cc1[R]</smiles>

1616

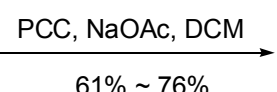<smiles>[R]c1cc2ccc3c4ccc([R])c([R])c4c(=O)n(C)c3c2cc1[R]</smiles> 


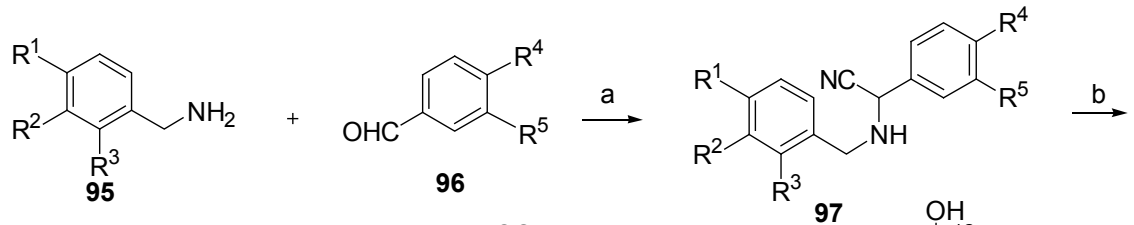

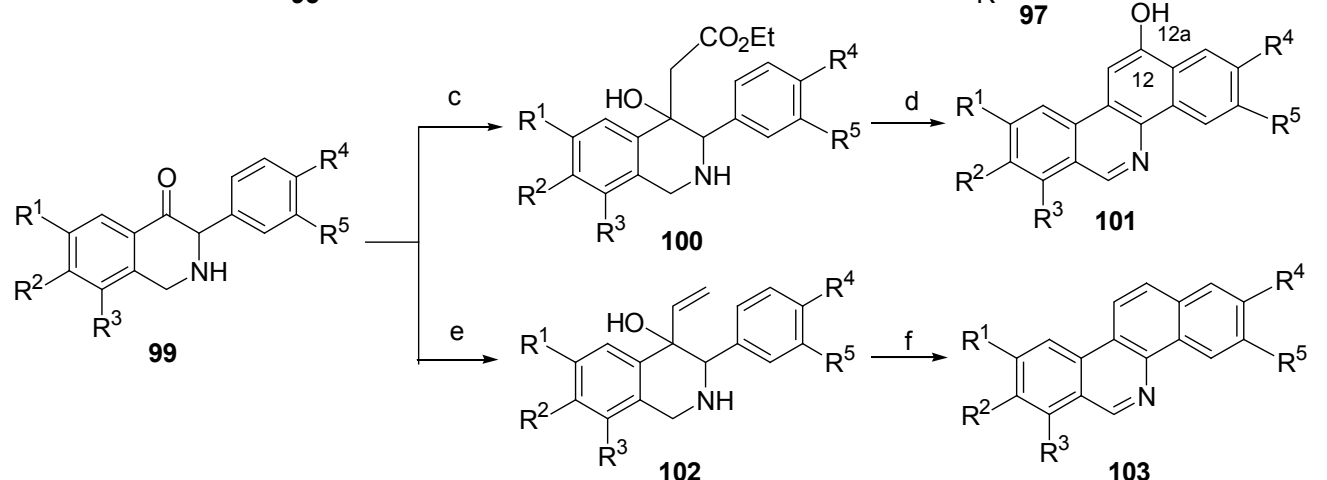

Reagents and conditions: (a) $\mathrm{KCN}, \mathrm{H}_{2} \mathrm{O} / \mathrm{C}_{2} \mathrm{H}_{5} \mathrm{OH}$, r.t., $91 \%$; (b) $\mathrm{HF}$, brine $15{ }^{\circ} \mathrm{C}, 100 \%$; (c) $\mathrm{BrCH}_{2} \mathrm{CO}_{2} \mathrm{Et}, \mathrm{Zn}$, dioxane/DMM, $80 \%$; (d) $\mathrm{H}_{2} \mathrm{SO}$, $78 \%$; (e) $\mathrm{CH}_{2}=\mathrm{CHMgBr}$, THF; (f) $\mathrm{MeSO}_{3} \mathrm{H}$

\section{Scheme 22}

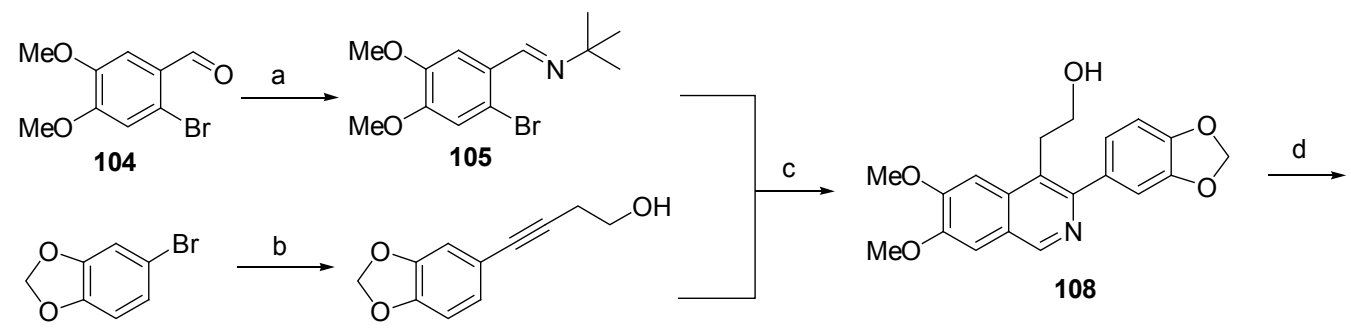

106

107<smiles>COc1cc2c(cc1OC)=CC(C)=c1ccc3cc4c(cc3c1=NC=2)OCO4</smiles>

Reagents and conditions: (a) tert-butylamine, 96\%; (b) $\mathrm{Pd}\left(\mathrm{PPh}_{3}\right)_{2} \mathrm{Cl}_{2}$, Cul, 3-butyn-1-ol, DIPA, 80\%; (c) $\mathrm{NeBr}_{2}(\mathrm{dppe}), \mathrm{Zn}, \mathrm{Na}_{2} \mathrm{CO}_{3}, \mathrm{DMF}, 73 \%$; (d) $(\mathrm{COCl})_{2}$, DMSO, DIPEA, DCM, $77 \%$; (e) $\mathrm{AcOH}, 40 \% \mathrm{HBr}, 78 \%$

Scheme 23
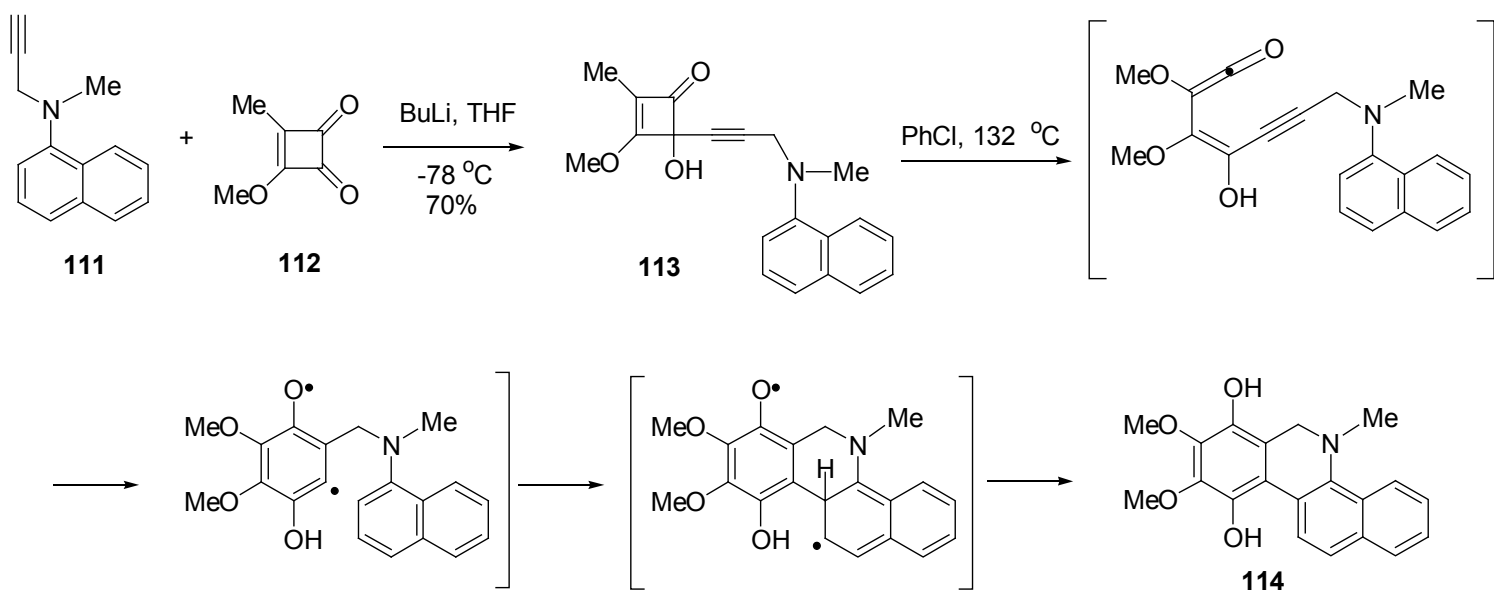

Scheme 24 
成路线仅需两步反应. 重排反应底物 113 由 $N$-炔甲基$\mathrm{N}$-甲基-1-䓺胺(111)与方酸二甲酯(dimethyl squarate, 112)反应得到. 以氯苯作为溶剂, 底物 113 在 $132{ }^{\circ} \mathrm{C}$ 下 重排即可转化为 5,6-二氢苯并啡定(114), 得率为 $45 \%$ (Scheme 24). Hergueta等将 113 中醇羟基氢氝代后, 证实 该热力学重排反应为自由基机理.

2005 年, Clement 等 ${ }^{[52]}$ 以商品化取代苯甲醛(115)和 2 equiv. 的邻甲基苯甲㲵(116)为原料, 在叔丁醇钾作用 下一步反应构建苯并啡啶母核基本骨架，同时能够在母 核骨架 11 位引入芳基(Eq. 5). 这也是迄今为止文献报道 的合成苯并啡啶母核最便捷的方法.

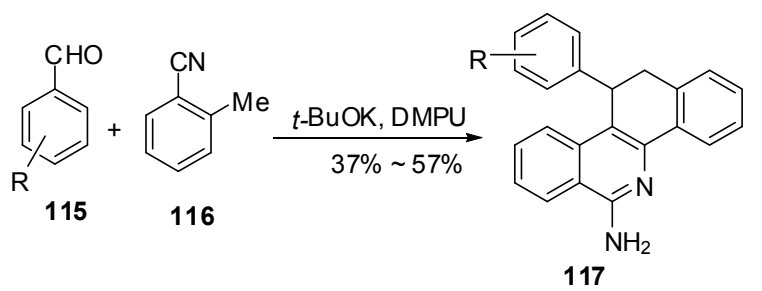

Clement 对可能的反应机理进行了探讨, 一分子邻 甲基苯甲㲵在叔丁醇钾的作用下与苯甲醛反应脱水得 到苯乙烯中间体 118, 另一分子邻甲基苯甲氰在碱性条 件下生成碳负离子对 118 中的双键加成后得到中间体 120. 中间体 120 中的一个亚甲基在碱性条件下失去质 子生成的碳负离子亲核加成分子中的氰基, $\mathrm{C}$ 环环合后 得到烯胺 121. 烯胺 $\mathrm{N}$ 原子作为亲核体加成另一氰基, $\mathrm{B}$ 环环合后得到目标产物 117 (Scheme 25).

苯甲醛中取代基 $\mathrm{R}$ 为供电子烷氧基或卤素原子时
对整个反应的得率没有影响 ${ }^{[53]}$. 但是以甲醛为原料时 目标产物 6-氨基-11,12-二氢苯并啡啶(118)的得率降低 至 $11 \%$, 影响 118 得率的原因仍然有待研究. 118 被叔丁 基亚硝酯氧化后得到 6- $O$-苯并啡啶(119)，随后还原羰 基，酸性条件下脱水得到全芳香化苯并啡啶(Scheme $26)^{[54]}$.

\section{4 总结与展望}

近年来，具有取代基多样性和高目标产物收率的苯 并啡定生物碱合成方法的建立为这类生物碱生物活性 和构效关系研究提供了便利. 特别是具有取代基兼容性 的钯催化多组分串联反应极大地提高了这类生物碱的 合成效率. 虽然部分合成方法具有反应步骤少，转化率 高等优点，但对反应底物取代基的性质有特殊的要求. 因此，在苯并啡啶生物碱生物活性研究过程中，我们可 依据所需要目标产物的亚结构类型或取代基类型来选 择合适的合成路线. 此外，在所有苯并啡定生物碱合成 方法中，仅 $\mathrm{C}_{11}-\mathrm{C}_{11 \mathrm{a}}$ 成键环合过程与 6-着基 protopine 向二氢苯并啡啶的转化过程有类似之处. 以天然 protopine 生物碱为反应底物的高效仿生化学合成将会 是苯并啡啶生物碱合成研究中一个非常有意思并且具 有挑战性的工作。当然，由于药用植物资源和天然合成 原料来源的有限性, 科学家们一直致力于采用全合成手 段获得苯并啡啶类生物碱，这些工作有效地推动了生物 碱合成化学的发展. 在上述研究工作的基础上, 苯并啡 啶类生物碱活性和合成方法研究有望成为生物碱研究 领域的一个重要研究方向.
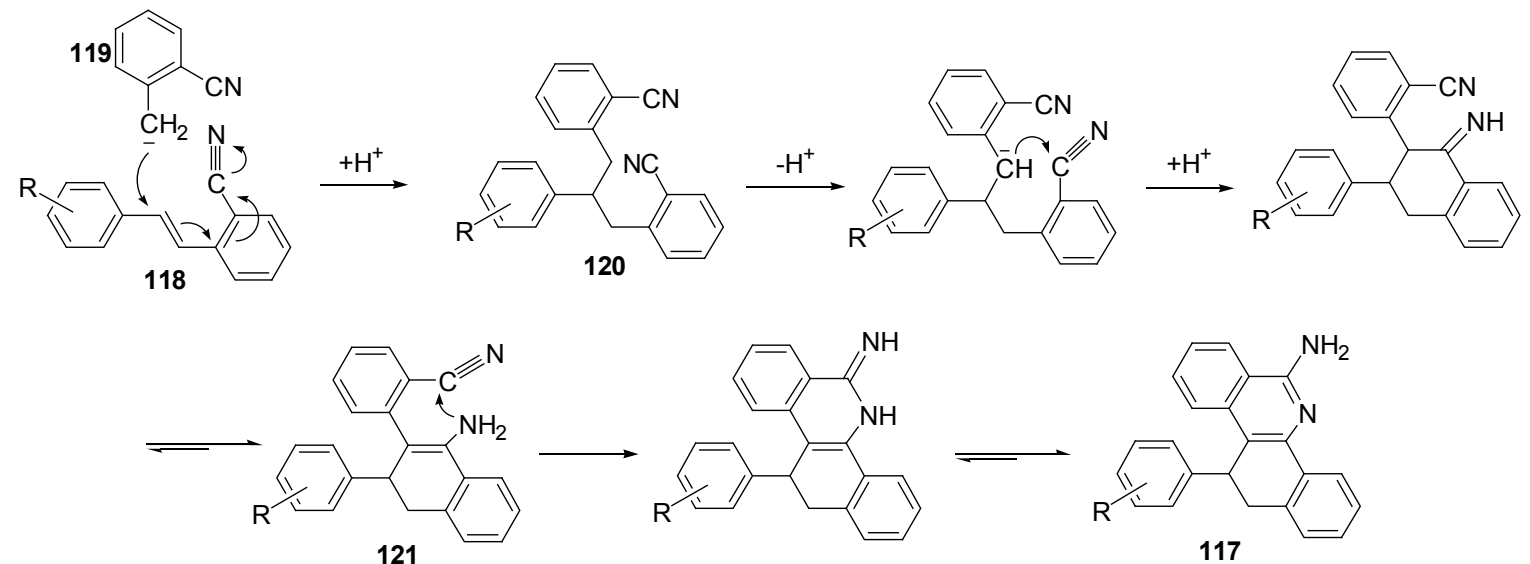

Scheme 25<smiles>Nc1nc2c(c3ccccc13)CCc1ccccc1-2</smiles>
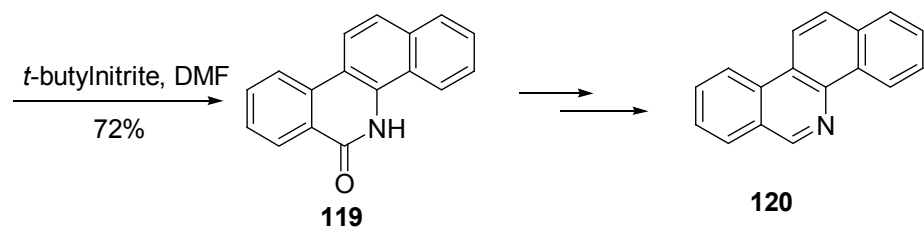

Scheme 26 


\section{References}

[1] Bentley, K. W. Nat. Prod. Rep. 2005, 22, 249.

[2] Dvořák, Z.; Kubáň, V.; Klejdus, B.; Hlaváč, J.; Vičar, J.; Ulrichová, J.; Šimánek, V. Heterocycles 2006, 68, 2403.

[3] Blank, R.; Muller-Siegwardt, B.; Wolffram, S. Livestock Sci. 2010, 134, 24.

[4] Newman, S. E.; Roll, M. J.; Harkrader, R. J. Hortscience 1999, 34 , 686.

[5] Newton, S. M.; Lau, C.; Gurcha, S. S.; Besra, G. S.; Wright, C. W. J. Ethnopharmacol. 2002, 79, 57.

[6] Mackay, S. P.; Meth-Cohn, O.; Waigh, R. D. Adv. Heterocycl. Chem. 1997, 67, 345.

[7] Ishikawa, T.; Ishii, H. Heterocycles 1999, 50, 627.

[8] Zenk, M. H. Pure Appl. Chem. 1994, 66, 2023.

[9] Morrison, G. C.; Cetenko, W.; Shavel Jr, J. J. Org. Chem. 1958, 23, 42.

[10] Ishii, H.; Ichikawa, Y. I.; Kawanabe, E.; Ishikawa, M.; Ishikawa, T.; Kuretani, K.; Inomata, M.; Hoshi, A. Chem. Pharm. Bull. 1985, 33, 4139.

[11] Satio, T.; Yoshida, M.; Ishikawa, T. Heterocycles 2001, 54, 437.

[12] Yoshida, M.; Watanabe, T.; Ishikawa, T. Heterocycles 2001, 54, 433.

[13] Ishikawa, T.; Shimooka, K.; Narioka, T.; Noguchi, S.; Saito, T.; Ishikawa, A.; Yamazaki, E.; Harayama, T.; Seki, H.; Yamaguchi, K. J. Org. Chem. 2000, 65, 9143.

[14] Treus, M.; Estevez, J. C.; Castedo, L.; Estevez, R. J. Tetrahedron Lett. 2000, 41, 6351.

[15] Vicario, J. L.; Badía, D.; Domínguez, E.; Carrillo, L. Tetrahedron: Asymmetry 2000, 11, 1227.

[16] Watanabe, T.; Ohashi, Y.; Yoshino, R.; Komano, N.; Eguchi, M. Org. Biomol. Chem. 2003, 1, 3024.

[17] Parenty, A.; Moreau, X.; Campagne, J. M. Chem. Rev. 2006, 106, 911.

[18] Yoshida, M.; Watanabe, T.; Ishikawa, T. Tetrahedron Lett. 2002, 43, 6751.

[19] Fleming, M. J.; McManus, H. A.; Rudolph, A.; Chan, W. H.; Ruiz, J.; Dockendorff, C.; Lautens, M. Chem. Eur. J. 2008, 14, 2112.

[20] Lautens, M.; Dockendorff, C. Org. Lett. 2003, 5, 3659.

[21] Ishihara, Y.; Azuma, S.; Choshi, T.; Kohno, K.; Ono, K.; Tsutsumi, H.; Ishizu, T.; Hbino, S. Tetrahedron 2011, 67, 1320.

[22] Kohno, K.; Azuma, S.; Choshi, T.; Nobuhiro, J.; Hibino, S. Tetrahedron Lett. 2009, 50, 590.

[23] Lysén, M.; Madden, M.; Kristensen, J. L.; Vedsø, P.; Zøllner, C.; Begtrup, M. Synthesis 2006, 3478.

[24] Bernardo, P. H.; Wan, K.; Sivaraman, T.; Xu, J.; Moore, F. K.; Hung, A. W.; Mok, H. Y. K.; Yu, V. C.; Chai, C. L. L. J. Med. Chem. 2008, 51, 6699 .

[25] Yapi, A.; Desbois, N.; Chezal, J.; Chavignon, O.; Teulade, J.; Valentin, A.; Blache, Y. Eur. J. Med. Chem. 2010, 45, 2854.
[26] Harayama, T.; Akiyama, T.; Akamatsu, H.; Kawano, K.; Abe, H.; Takeuchi, Y. Synthesis 2001, 444.

[27] Harayama, T.; Akiyama, T.; Nakano, Y.; Shibaike, K.; Akamatsu, H.; Hori, A.; Abe, H.; Takeuchi, Y. Synthesis 2002, 237.

[28] Harayama, T.; Akamatsu, H.; Okamura, K.; Miyagoe, T.; Akiyama, T.; Abe, H.; Takeuchi, Y. J. Chem. Soc., Perkin Trans. 1 2001, 523.

[29] Ramani, P.; Fontana, G. Tetrahedron Lett. 2008, 49, 5262.

[30] Nakanishi, T.; Suzuki, M.; Mashiba, A.; Ishikawa, K.; Yokotsuka, T. J. Org. Chem. 1998, 63, 4235.

[31] Nakanishi, T.; Suzuki, M. S. Org. Lett. 1999, 7, 985.

[32] Budén, M. E.; Rossi, R. A. Tetrahedron Lett. 2007, 48, 8739.

[33] Moreno, I.; Tellitu, I.; Etayo, J.; SanMartín, R.; Dmínguez, E. Tetrahedron 2001, 57, 5403.

[34] Konno, F.; Ishikawa, T.; Kawahata, M.; Yamaguchi, K. J. Org. Chem. 2006, 71, 9818.

[35] Ito, M.; Konno, F.; Kumamoto, T.; Suzuki, N.; Kawahata, M.; Yamaguchi, K.; Ishikawa, T. Tetrahedron 2011, 67, 8041.

[36] Lv, P.; Huang, K.; Xie, L.; Xu, X. Org. Biomol. Chem. 2011, 9, 3133.

[37] Rayabarapu, D. K.; Shukla, P.; Cheng, C.-H. Org. Lett. 2003, 5, 4903.

[38] Madan, S.; Cheng, C.-H. J. Org. Chem. 2006, 71, 8312.

[39] Blanchot, M.; Candito, D. A.; Larnaud, F.; Lautens, M. Org. Lett. 2011, 13, 1486.

[40] Candito, D. A.; Lautens, M. Angew. Chem. 2009, 121, 6841.

[41] Cho, W. J.; Park, M. J.; Imanishi, T.; Chung, B. H. Chem. Pharm. Bull. 1999, 47, 900.

[42] Le, T. N.; Gang, S. G.; Cho, W. J. Tetrahedron Lett. 2004, 45, 2763.

[43] Nguyen, T. Cho, W. J. Chem. Pharm. Bull. 2006, 54, 476.

[44] Le, T. N.; Gang, S. G.; Cho, W. J. J. Org. Chem. 2004, 69, 2768.

[45] Le, T. N.; Van, H. T. M.; Lee, S. H.; Choi, H. J.; Lee, K. Y.; Kang, B. Y.; Cho, W. J. Arch. Pharm. Res. 2008, 31, 6.

[46] Vivario, J. L.; Badía, D.; Dominguea, E.; Crespo, A.; Carrillo, L. Tetrahedron: Asymmetry 1999, 10, 1947.

[47] Lynch, M. A.; Duval, O.; Sukhanova, A.; Devy, J.; MacKay, S. P.; Waigh, R. G.; Nabiev, I. Bioorg. Med. Chem. Lett. 2001, 11, 2643.

[48] Vanquelef, E.; Amoros, M.; Boustie, J.; Lynch, M. A.; Waigh, R. D. J. Enzyme Inhib. Med. Chem. 2004, 19, 481.

[49] Nyangulu, J. M.; Hargreaves, S. L.; Sharples, S. L.; Mackay, S. P.; Waigh, R. D.; Duval, O.; Mberu, E. K.; Watkins, W. M. Bioorg. Med. Chem. Lett. 2005, 15, 2007.

[50] Luo, Y.; Mei, Y.; Zhang, J.; Lu, W.; Tang, J. Tetrahedron 2006, 62, 9131.

[51] Hergueta, A. R.; Moore, H. W. J. Org. Chem. 1999, 64, 5979.

[52] Clement, B.; Weide, M.; Wolschendorf, U.; Kock, I. Angew. Chem., Int. Ed. 2005, 44, 635.

[53] Kock, I.; Heber, D.; Weide, M.; Wolschendorf, U.; Clement, B. J. Med. Chem. 2005, 48, 2772.

[54] Kock, I.; Clement, B. Synthesis 2005, 1052.

(Qin, X.) 